\title{
A comprehensive review on privacy preserving data mining
}

\author{
Yousra Abdul Alsahib S. Aldeen ${ }^{1,2^{*}}$, Mazleena Salleh ${ }^{1}$ and Mohammad Abdur Razzaque
}

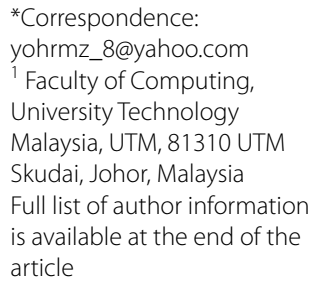

\begin{abstract}
Preservation of privacy in data mining has emerged as an absolute prerequisite for exchanging confidential information in terms of data analysis, validation, and publishing. Ever-escalating internet phishing posed severe threat on widespread propagation of sensitive information over the web. Conversely, the dubious feelings and contentions mediated unwillingness of various information providers towards the reliability protection of data from disclosure often results utter rejection in data sharing or incorrect information sharing. This article provides a panoramic overview on new perspective and systematic interpretation of a list published literatures via their meticulous organization in subcategories. The fundamental notions of the existing privacy preserving data mining methods, their merits, and shortcomings are presented. The current privacy preserving data mining techniques are classified based on distortion, association rule, hide association rule, taxonomy, clustering, associative classification, outsourced data mining, distributed, and k-anonymity, where their notable advantages and disadvantages are emphasized. This careful scrutiny reveals the past development, present research challenges, future trends, the gaps and weaknesses. Further significant enhancements for more robust privacy protection and preservation are affirmed to be mandatory.
\end{abstract}

Keywords: Privacy preserving, Data mining, Distortion, Association, Classification, Clustering, Outsourcing, K-anonymity

\section{Background}

Supreme cyberspace protection against internet phishing became a necessity. The intimidation imposed via ever-increasing phishing attacks with advanced deceptions created a new challenge in terms of mitigation. Lately, internet phishing caused significant security and economic concerns on the users and enterprises worldwide. Diversified communication channels via internet services such as electronic commerce, online-banking, research, and online trade exploiting both human and software vulnerabilities suffered from tremendous financial loss. Therefore, enhanced privacy preserving data mining methods are ever-demanding for secured and reliable information exchange over the internet. The dramatic increase of storing customers' personal data led to an enhanced complexity of data mining algorithm with significant impact on the information sharing. Amongst several existing algorithm, the Privacy Preserving Data Mining (PPDM) renders excellent results related to inner perception of privacy preservation and data mining. Truly, the privacy must protect all the three mining aspects including association

(c) 2015 Aldeen et al. This article is distributed under the terms of the Creative Commons Attribution 4.0 International License (http://creativecommons.org/licenses/by/4.0/), which permits unrestricted use, distribution, and reproduction in any medium, provided you give appropriate credit to the original author(s) and the source, provide a link to the Creative Commons license, and indicate if changes were made. 
rules, classification, and clustering (Sachan et al. 2013). The problems faced in data mining are widely deliberated in many communities such as the database, the statistical disclosure control and the cryptography community (Nayak and Devi 2011). The emergence new cloud computing technology allowed the business collaborators to share the data and supply the information for the mutual benefits. All of these are related to the cumulative capability to store users' individual data together with the rising complexity of data mining algorithms that affects the information exchange. Yet, the concepts, utilization, categorization, and various attributes of PPDM in terms of its strength and weakness are not methodically reviewed.

Currently, several privacy preservation methods for data mining are available. These include $K$-anonymity, classification, clustering, association rule, distributed privacy preservation, $L$-diverse, randomization, taxonomy tree, condensation, and cryptographic (Sachan et al. 2013). The PPDM methods protect the data by changing them to mask or erase the original sensitive one to be concealed. Typically, they are based on the concepts of privacy failure, the capacity to determine the original user data from the modified one, loss of information and estimation of the data accuracy loss (Xu and Yi 2011). The basic purpose of these approaches is to render a trade-off among accuracy and privacy. Other approaches that employ cryptographic techniques to prevent information leakage are computationally very expensive (Ciriani et al. 2008). Conversely, PPDMs use data distribution and horizontally or vertically distributed partitioning through multiple entities.

Sometimes the individuals are reluctant to share the entire data set and may wish to block the information using varieties of protocols. The main rationale for implementing such techniques is to maintain individuals' privacy while deriving collective results over the entire data (Aggarwal and Yu 2008). Despite much research a method with satisfactory privacy settings are far from being achieved. It is essential to protect the data information before it gets distributed to multi-cloud providers. To protect the privacy, clients' information must be identified prior to sharing with those unknown users not directly allowed to access the relevant data. This can be achieved by deleting from the dataset the unique identity fields such as name and passport number. Despite this information removal, there are still other types of information including date of birth, zip code, gender, number of child, number of calls, and account numbers which can be used for possible subjects' identification. Intensified and extensively robust privacy preservation measures in data mining must be implemented to prevent such types of breaching.

This presentation underscores the significant development of privacy preserving data mining methods, the future vision and fundamental insight. Several perspectives and new elucidations on privacy preserving data mining approaches are rendered. Existing literatures are systematically subcategorized to identify the strengths, gap, and weakness of various approaches. The paper is organized as follows. "Privacy preserving data mining" discusses in detail the requirement of privacy preserving data mining scheme in the context of internet phishing mitigation. The notable advantages and disadvantages of the existing methods are highlighted in "Shortcomings of PPDM methods". This section primarily focused on the creation of awareness and relevant action to be taken by all relevant quarters to protect privacy in secured data transfer over the web. "Conclusion" concludes the paper with further outlook in this field. 


\section{Differential privacy model}

Recently, differential privacy model is widely explored to render maximum security to the private statistical databases by minimizing the chances of records identification. There are several trusted party that holds a dataset of sensitive information such as medical records, voter registration information, email usage, and tourism. The primary aim is to providing global, statistical information about the data publicly available, while protecting those users privacy whose information is contained in the dataset. The concept of "indistinguishability" also called "differential privacy" signifies the "privacy" in the context of statistical databases. Generally, data privacy is viewed as a characteristic or annotation to data safety. Obviously, this view is incorrect because the objectives of the two domains are opposite. Conversely, security protects the data against unauthorized access when transmitted across a network. However, upon arriving to an authorized user no additional constraints are imposed on the data security to revealing the personal information of an individual. Thus, it is worth to determine the correlation between data security and data privacy because the former is prerequisite of the latter.

Data must be protected at storage and the transmission must be made via data security protocols. Moreover, in case data privacy is a goal, then some other steps must be considered to protect individuals confidentiality embodied in the data. It is important to describe the process of PPDM addresses in terms of data sharing and the results of data mining operation between a number of users $u_{1}, \ldots u_{m}$ with $m \geq 2$. The data is viewed as a database of $n$ records, each consisting of $l$ fields, where each record represents an individual $i_{i}$ and illustrates them through its fields. In a simplified representation a table $T$ contains rows to signify $i_{1}, \ldots i_{n}$ and columns that symbolizes the fields $a_{1}, \ldots a_{l}$. Assuming a fixed representation, each individual is represented by a vector of components $a_{1}, \ldots a_{l}$. The most useful dimension in PPDM is the protected privacy embedded in $T$, which an attacker wants to acquire. The other practical dimension is the possessive data structure, which belongs to one entity and need to be shared with another $(\mathrm{m}=2)$. It may be built from parts owned by different entities.

It is important to introduce some definitions to strengthen the PPDM concepts. Especially, an explicit identifier is an attribute that permits a direct connection of an instance (a row in $\mathrm{T}$ ) to a user $i$. For example, by identifying a cellular phone number or a driver's license number it may unambiguously connect the row in $T$, where this explicit identifier to a person $i$ is embedded. Conversely, a quasi-identifier being a set of individuals' non-explicit attributes may also link a row in $T$ to a specific person. For instance, in the United States the quasi-identifier triplet < date of birth, 5 digit postal code, gender> uniquely identifies $87 \%$ of the nation's population (Sweeney 2002). By combining a public healthcare information dataset with a publicly available voters' list and using quasi-identifiers, Sweeney convinced that it is possible to mine the secret health records of all state employees from a published dataset of the Massachusetts governor, where only explicit identifiers is removed. Generally, the primary PPDM identity protection methods that are drawn on simple ideas are known to people as they are abundantly accessible in the literatures and films. These concepts are portrayed as "hiding in the crowd" and "camouflage". One of the "hiding in the crowd" approach to data privacy is the k-anonymity. Actually, the k-anonymity method (Sweeney 2002; Nergiz et al. 2009) modifies the original data $T$ to obtain $T^{\prime}$ such that for any quasi-identifier $q$ that can 
be built from attributes of $T$ there are at least $k$ instances in $T^{\prime}$ so that q matches these instances. Moreover, datasets require generalization to satisfy $k$-anonymity.

\section{Privacy preserving data mining}

Recently, the relevance of privacy-preserving data mining techniques is thoroughly analyzed and discussed by Matwin (2013). Utilization of specific methods revealed their ability to preventing the discriminatory use of data mining. Some methods suggested that any stigmatized group must not be targeted more on generalization of data than the general population. Vatsalan et al. (2013) reviewed the technique called 'PrivacyPreserving Record Linkage' (PPRL), which allowed the linkage of databases to organizations by protecting the privacy. Thus, a PPRL methods based taxonomy is proposed to analyse them in 15 dimensions. Qi and Zong (2012) overviewed several available techniques of data mining for the privacy protection depending on data distribution, distortion, mining algorithms, and data or rules hiding. Regarding data distribution, only few algorithms are currently used for privacy protection data mining on centralized and distributed data. Raju et al. (2009) acknowledged the need to add or to multiply the protocol based homomorphic encryption along with the existing concept of digital envelope technique in obtaining collaborative data mining while keeping the private data intact among the mutual parties. The proposed technique exhibited considerable influence on different applications.

Malina and Hajny (2013) and Sachan et al. (2013) analysed the current privacy preserving solutions for cloud services, where the solution is outlined based on advanced cryptographic components. The solution offered the anonymous access, the unlink ability and the retention of confidentiality of transmitted data. Finally, this solution is implemented, the experimental results are obtained and the performance is compared. Mukkamala and Ashok (2011) compared a set of fuzzy-based mapping methods in the context of privacy-preserving characteristics and the capability to maintain the same connection with other fields. This comparison is subjected to: (1) the four front modification of the fuzzy function definition, (2) the introduction of the seven ways to join different functional values of a particular data item to a single value, (3) the utilization of several similarity metrics for the comparison of the original data and mapped data, and (4) the evaluation of the influence of mapping on the derived association rule.

\section{Data distortion dependent PPDM}

Kamakshi (2012) proposed a novel idea to dynamically identify the sensitive attributes of PPDM. Identification of these attributes depends on the threshold limit of sensitivity of each characteristic. It is observed that the data owner modified the value under identified sensitive attributes using swapping technique to protect the privacy of sensitive information. The data is modified in such a manner that the original properties of the data remain unchanged. Despite the novelty it remains time expensive. Subsequently, Zhang et al. (2012a) introduced a newly enhanced historical probability based noise generation strategy called HPNGS. The simulation results confirmed that the HPNGS is capable in reducing the number of noise requirements over its random complement as much as $90 \%$. Later, they focused on the privacy protection and noise obfuscation in cloud computing (Zhang et al. 2012b). Consequently, a novel association probability 
based noise generation strategy (APNGS) is developed. The analysis confirmed that the proposed APNGS significantly improved the privacy protection on noise obfuscation involving association probabilities at a reasonable extra cost than standard representative strategies.

Li et al. (2009a) presented a low-cost and less risky anonymous perturbation technique via homomorphism encryption and anonymous exchange. The proposed technique displayed robustness for optimized parameters. It is complex, loss in utility of data. Kamakshi and Babu (2010) introduced three models including clients, data centres, and database in every site. The data centre is completely passive, so that the clients and the site database role appear exchangeable. Islam and Brankovic (2011) proposed an architecture involving different novel techniques that affected all the attributes in the database. Experimental findings showed that the proposed architecture is very efficient in preserving the original patterns in a perturbed dataset. Wang and Lee (2008) introduced a technique to prevent Forward-Inference Attacks, in the sanitized data (implies original data) created by the sanitization.

\section{Association rule based PPDM}

An improved distortion technique for privacy preserving frequent item-set mining is proposed by Shrivastava et al. (2011), where two probability parameters (fp and nfp) are employed. Better accuracy is achieved in the presence of a minor reduction in the privacy by tuning these two parameters. Furthermore, this algorithm produced the optimum results when the fraction of frequent items among all the available items is less. PPDM is used in various fields for its enhanced efficiency and security. Presently, it is facing a rule mining challenge. Vijayarani et al. (2010a) explained the techniques of statistical disclosure control community, the database community, and the cryptography community. Less utility of data requires high cost. Aggarwal and Yu (2008) emphasized two significant factors involving the association rule mining such as confidence and support. For an association rule $\mathrm{X}=>\mathrm{Y}$, the support is the percentage of transactions in the dataset which includes X U Y. The confidence (also called strength) of an association rule $\mathrm{X}=>\mathrm{Y}$ is the ratio of the transactions number by $\mathrm{X}$. Furthermore, Belwal et al. (2013) reduced the basis of support and confidence of sensitive rules without modifying directly the given database. However, alteration can indirectly be performed via newly incorporating parameters associated to database transactions and association rules. New additions include $\mathrm{M}$ support (modified support), $\mathrm{M}$ confidence (modified confidence) and Hiding counter. The algorithm utilized the definition of support and confidence. Thus, it hided the required sensitive association rule without any side effect. However, it can hide only the rules for single sensitive item on the LHS.

Jain et al. (2011) developed a new algorithm to enhance and reduce the support of the LHS and RHS rule item to hide or secure the association rules. The proposed algorithm is found to be advantageous as it made minimum modification to the data entries to hide a set of rules with lesser CPU time than the previous work. It is limited to association rule only. Naeem et al. (2010) proposed an architecture which screened the restricted association rules with complete removal of the known side effects such as the generation of unwanted, non-genuine association rules while yielding no 'hiding' failure. In this architecture, standard statistical measures are used instead of conventional framework 
of support and confidence to create association rules, particularly weighing procedure based on central tendency. Li and Liu (2009) introduced an association rule mining algorithm for privacy preserving known as DDIL. The proposed algorithm is based on inquiry limitation and data disturbance. The original data can be hidden or disturbed by using DDIL algorithm to improve the privacy efficiently. This is an effective technique to generating frequent items from transformed data. Experimental results displayed that the proposed technique is efficient to generating acceptable values of privacy balance with suitable selection of random parameters.

\section{Hide association rule based PPDM}

Fast Hiding Sensitive Association Rules (FHSAR) algorithm is introduced by Weng et al. (2008). This secured the SAR with fewer side effects, where a strategy is established to avoid hidden failures. Besides, two heuristic techniques are developed to improve the efficiency of the system to solve the problems. The heuristic function is further utilized to determine the earlier weight for each particular transaction so that the order of modified transactions can be decided efficiently. Consequently, the connection between the sensitive association rules and each transaction in the original database are analyzed by successfully choosing the suitable item for modification. The efficient sanitization of sensitive information for updated database need to be studied. Dehkordi et al. (2009) presented a new multi-objective technique to hide the sensitive association rules and to enhance the security of database. In fact, this maintained the utility and of mined rules at efficient level. The proposed algorithm is based on genetic algorithm (GA) concept, where the privacy and accuracy of dataset are enhanced. Gkoulalas-Divanis and Verykios (2009) developed an exact border-based technique to obtain an optimal solution to hide sensitive frequent item sets with minimum extension of the original database generated synthetically via the database extension. This is accomplished via the following: (1) by formulating the generation of the database extension as a constraint satisfaction problem, (2) using mapping of the constraint satisfaction issues to an equivalent binary integer programming problem, (3) via the manipulation of underutilized synthetic transactions to increase the support of non-sensitive item sets, (4) employing the minimally relaxing constraint satisfaction problem to offer an approximate solution close to the optimal one when an ideal solution does not exist, and (5) by partitioning the universe of the items to enhance the efficiency of the proposed hiding algorithm.

Li et al. (2009b) proposed a new algorithm to sanitize a transactional database. This is item-set oriented, where the support of large item-sets are considerably reduced below the threshold defined by the client. Thus, no rules can be obtained from the specific item-sets. A new technique is also introduced to select the items that required removal from the dataset to avoid the detection of a set of rules. The main limitations are associated with the selection of victim-items without affecting the non-sensitive patterns when the sanitization of 3rd and the 4th sensitive transactions are defined. Kasthuri and Meyyappan (2013) presented a new technique to identify the sensitive items by hiding the susceptible association rules. The proposed technique located the frequent item sets and produced the association rules. Representative association rules concept is employed to detect the sensitive items. Hiding the sensitive association rules using selected sensitive items is worth looking. Quoc et al. (2013) have developed heuristic algorithm based 
on the intersection lattice of frequent item-sets to secure the set of sensitive association rules employing distortion method. To reduce the side effects, the heuristic for confidence and support reduction based on intersection lattice (HCSRIL) algorithm are used. This specified the victim item and reduced the number of transactions by causing least impact on item-sets variations in Gen(FI). In addition, Domadiya and Rao (2013) introduced a heuristic based algorithm called Modified Decrease Support of RHS item of Rule Clusters (MDSRRC) to secure the delicate association rules using multiple items in consequent (RHS) and antecedent (LHS). This algorithm successfully addressed the drawbacks of existing rule hiding DSRRC algorithm. Experimental findings revealed the efficiency and capability of the proposed algorithm to maintaining the database quality. By minimizing the modifications on database the efficiency can be enhanced with reduced side effects.

\section{Classification based PPDM}

Xiong et al. (2006) proposed a closet neighbour classification method based on SMC techniques to resolve the privacy challenges in few stages including the pf selection of the privacy preserving closet neighbour and the categorization of privacy preserving. The proposed algorithm is balanced in terms of accuracy, performance, and privacy protection. Furthermore, it is adaptable to the various settings to fulfilling different optimization condition. Singh et al. (2010) provided a simple and efficient privacy preserving classification for cloud data. Jaccard similarity measure is used to compute the nearest neighbours for $K$-NN classification and the equality test is introduced to compute it between two encrypted records. This approach facilitated a secured local neighbour computation at each node in the cloud and classified the unseen records via weighted $K-\mathrm{NN}$ classification scheme. It is significant to focus on enabling the robustness of the presented approach so that generalization to multiple data mining tasks can be made, where security and privacy are needed.

Baotou (2010) introduced an efficient algorithm based on random perturbation matrix to protect privacy classification mining. It is applied on discrete data of character type, Boolean type, classification type and number types. The experimental revealed the significantly enhanced features of proposed algorithm in terms of privacy protection and accuracy of mining computation, where the computation process is greatly simplified but at higher cost. Vaidya et al. (2008) developed an approach for vertically partitioned mining data. This technique could modify and extend a variety of data mining applications as decision trees. More efficient solutions are needed to find tight upper bound on the complexity. Kantarcioglu and Vaidya (2003) emphasized the use of secure logarithm and summation, where the distributed naive Bayes classifier are securely determined. The experimental results strongly supported the concept of few useful protected protocols that facilitated the secure deployment of different types of distributed data mining algorithms. The classification of privacy preserving methods and standard algorithms for each class is reviewed by Sathiyapriya and Sadasivam (2013), where the merits and limitations of different methods are exemplified. The optimal sanitization is found to be NP-Hard in the presence of privacy and accuracy trade-off. 


\section{Clustering based PPDM}

Yi and Zhang (2013) overviewed various earlier solutions to preserve privacy of distributed k-means clustering and provided a formal definition for equally contributed multiparty protocol. An equally contributed multiparty k-means clustering is applied on vertically partitioned data, wherein each data site contributed k-means clustering evenly. According to basic concept, data sites collaborated to encrypt $k$ values (each associated to a distance between the centre and point) with a common public key in each step of clustering. Then, it securely compared $k$ values and outputted the index of the minimum without displaying the intermediate values. In some setting, this is practical and more efficient than Vaidya-Clifton protocol (Vaidya et al. 2008).

\section{Associative classification based PPDM}

An associative classification model based on vertically partitioned datasets is introduced by Raghuram and Gyani (2012). A scalar product based third party privacy preserving model is adopted to preserve the privacy for data sharing process between multiple users. The accuracy of the presented method is authenticated on its VCI databases with inspiring results. Lin and Lo (2013) presented a set of algorithms comprising of Equal Working Set (EWS), Small Size Working Set (SSWS), Request on Demand (ROD) and the Progressive Size Working Set (PSWS). This repeated mining offered a scalable, fast and reliable service for different-tasks on computing environments. The presented algorithms demonstrated an outstanding efficiency in terms of scalability and execution time under different simulation conditions. Although CARM is a fast and scalable distributed algorithm in comparison with previous studies, the scalability is still limited. This is because the HD-Mine used in CARM establishes the FP-tree in the main memory of the trusted node. In the absence of any memory space to mine the conditional FP-tree in the trusted node, the reconstructed conditional FP-tree is distributed to an available computing node for mining. The trusted node must provide sufficient memory space for the original FP-tree. Clearly, the scalability is restricted by the major memory size of the trusted node.

Harnsamut and Natwichai (2008) developed a novel heuristic algorithm based on Classification Correction Rate (CCR) of particular database to secure the privacy and sustain the quality of data. The proposed algorithm is tested and the experimental results are validated. The heuristic algorithm is found to be highly effective and efficient. Seisungsittisunti and Natwichai 2011) highlighted the issues related to data transformation to protecting privacy for data mining technique and associative classification in an incremental-data scenario. An incremental polynomial-time algorithm is proposed to transform the data to maintain a privacy standard called k-anonymity. Quality can still be maintained even under transformation when constructing an associative classification model. Different experiments are performed to evaluate developed algorithm performance and compared with non-incremental algorithm. It is established to be more efficient in every problem setting. It is worth to examine the stored data in the distributed systems rather than a single repository. 


\section{Privacy preserving outsourced data mining}

Giannotti et al. (2013) explained the issues involving the outsourcing of association rule mining task for a corporate privacy-preserving network. An attack model is developed based on the background knowledge for privacy preserving outsourced mining. An encryption scheme, known as Rob Frugal is proposed. This is based on 1-1 substitution ciphers of items, which included the fake transactions to share each cipher item with the same frequency as $\geq k-1$ to the others. A compact synopsis of the fake transactions is used for true support of mined patterns from which the server can be recovered efficiently. It is demonstrated that the proposed scheme is robust against adversarial attack which is based on the actual items and their exact support. This framework assumed that the attacker is unaware of such information. Furthermore, any relaxation may break our encryption scheme and bring privacy vulnerabilities. They investigated encryption schemes that could resist such privacy vulnerabilities. The strategies for the improvement of the RobFrugal algorithm to minimize the number of spurious patterns are also explored.

Worku et al. (2014) enhanced efficiency of the above scheme by reducing the computational intensive operations such as bilinear mapping. The scheme revealed secure and efficient results after a detailed analysis on security performance. However, the data block insertion made the proposed scheme non-dynamic. Thus, the development of a fully dynamic and secure public auditing scheme remains an open challenge for a cloud system. Arunadevi and Anuradha (2014) investigated the issues related to outsourcing of frequent item-sets for a corporate privacy preserving architecture. An attack model is introduced by considering that the attackers are fully aware of the items and support of the item. In addition, even in the eventuality the attackers are totally conscious of the details of the encryption algorithm and some pairs of item with the corresponding cipher values. These basic assumptions remarkably improved the security of the system and eliminated the item and item-set based attack as well as reduced the processing time.

Lai et al. (2014) proposed the first semantically secured solution for outsourcing association rule mining with data privacy, mining privacy and soundness. These solutions are non-deterministic and secured against an adversary at cloud servers. It is capable to adaptively obtaining plaintext-cipher text pairs as required by semantic security. The adversary may also insert false data into the data mining results. In comparison, adversary models used in previous works on outsourcing association rule mining assumed that the honesty of adversary/server but remained curious. It is not capable to obtaining any plaintext-cipher text pairs in attacks. Consequently, the sub-situation mappings based solutions are neither semantically secured nor ensured the soundness for the data mining results. Kerschbaum and Julien (2008) presented a searchable encryption scheme for outsource data analysis. In this scheme the client had to encrypt the data only once and transmit the encrypted information to the data analyst. The data analyst conducted a number of queries for required permission from the client to translate the data contents in the queries. The proposed encryption schemes permitted the search of keyword and range queries. The scheme also allowed queries to reprocess the output of earlier queries as tokens to make dependent queries without interface. The proposed scheme is found to be secured. There are many open questions in the area of search-able 
encryption. In case of outsourced data analytics, it is most interesting to combine the efficiency improvements possible for range queries with the necessary security requirements via pairing-based cryptography.

\section{Distributed method based PPDM}

Ying-hua et al. (2011) surveyed the Distributed Privacy Preserving Data Mining (DPPDM) depending on different underlying technologies. Existing techniques are categorized into three groups such as (1) secure multi-party computation, (2) perturbation and (3) restricted query. Li (2013) elucidated the advantages and drawbacks of each method by developing and analyzing a symmetric-key based privacy-preserving scheme to support mining counts. An incentive consideration is proposed to the study the secure computation by presenting a reputation system in wireless network. The proposed system offered an incentive for misbehaving nodes to behave properly. Experimental results revealed the system effectiveness in detecting the misbehaving nodes and enhancing the average throughput in the whole network. Furthermore, Dev et al. 2012) acknowledged the privacy risks related to data mining on cloud system and presented a distributed framework to remove such risks. The proposed approach involved classification, disintegration, and distribution. This avoided the data mining by preserving the privacy levels, splitting the data into chunks and storing them into suitable cloud providers. Though, the proposed system offered a suitable way to safe privacy from mining based attacks, but it added a performance overhead as client accessed the data frequently. For instance, client had to run a global data analysis for a complete dataset, where the analysis required accessing the data through different locations with a degraded performance.

Tassa (2014) developed a protocol for secured mining of association rules in horizontally distributed database. The proposed protocol possessed advantages over leading protocols in terms of performance and security. It included two set of rules including (1) a multi-party protocol to compute the union or intersection of private subsets possessed by each client and (2) a protocol to test the presence of an element held by client in a subset held by another. Techniques based on Field and Row-Level distribution of transactional data are proposed by Chan and Keng (2013). They presented a distributed framework to preserve outsourcing association mining rules and explored the possibility of its deployment. Database information based on its characteristics is distinguished for the distribution to multiple servers. Its privacy notions are examined from two separate viewpoints such as distribution of support values and K-anonymity. The proposed algorithms for allocating transactions to outsourced servers are based on the importance of the types of privacy notion to a user. Dong and Kresman (2009) explained the relation between distributed data mining and prevention of indirect disclosure of private data in privacy preserving algorithms, where two protocols are devised to avoid such disclosures. The first one was a simple add-on to a protocol used for different application, whereas the second one provided the suitability of collusion resistance and fewer broadcasts. The simplicity of the proposed protocols enabled minimal requirements for computation, easy data storage or data structures. Consequently, the notion of trust is introduced and the performance of certain ID assignment protocols is addressed.

Aggarwal et al. (2005) discussed data encryption based methods, which caused a large overhead in query processing. A new distributed framework is proposed to enable 
privacy-preservation for the outsourced storage of data. Different techniques are used to decompose the data. It demonstrated improved queries when implemented in such types of distributed system. A new definition for privacy is coined based on hiding sets of attributes. It discussed the secured privacy achievement of the proposed decomposition approaches and identified the best privacy-preserving decomposition technique. Other future work includes identifying improved algorithms for decomposition, expanding the scope of techniques available for decomposition (supporting replication, and incorporation of these techniques into the query optimization framework). Xu and Yi (2011) investigated the privacy-preserving distributed data mining that passed through different stages and persisted. Taxonomy is proposed to endorse the standardization and assessment of the protocols efficiency. This might be applied to categorize such PPDDM protocols based on predefined dimensions. The dimensions included the data partitioning model, mining algorithms, privacy preservation methods and secured communication model. This area is prospective. Yet, the solution and evaluation work is still open for further investigation.

Inan and Saygin (2010) presented a technique to assemble dissimilarity matrix for horizontal distributed data mining. The comparison required all the record operations in the form of pair for personal private datasets which are distributed horizontally to different sites. This approach considered the data either in the form of character or numerical. For these two different types of data sets, a number of comparison functions are made available. However, as expected, ensuring privacy has its costs, considering the comparison against the baseline protocol where private data is shared with third parties. We used the secured comparison protocols for clustering horizontally partitioned datasets. There are various other application areas of these methods such as record linkage and outlier detection problems Nanavati and Jinwala (2012) elaborated different approaches used to find global and partial cycles in a distributed setup while keeping the privacy of the particular parties secured in a co-operative setup. The interleaved algorithm is extended and modified to determine global cycles in cyclic association rules privately. The privacy preservation techniques are recommended on the basis of homomorphic approach and secret sharing. It is concluded that the approaches based on Shamir's secret sharing can be employed to detect the partial global cycles. However, few open research challenges including the application of these privacy preserving theories to other temporal rule mining methods like calendric association rules and temporal predicate association rules need to be addressed. Another research challenge also involves deciphering the most efficient and accurate technique in this scenario by practically comparing the cost for each method.

Agrawal and Srikant (2000) developed a uniform randomization method based association rule for the categorical datasets. In this approach, before sending a data to server, the client is replaced each item by a new item which is originally absent in the data. The substitution process of specific values from datasets with other values is called uniform randomization. This is a generalization of the Warner's "randomized response" technique. In other types of data reconstruction techniques the original data are put aside and are initiated via sanitizing known as "knowledge base". Thus, newly obtained data is then reassembled based on the sanitized knowledge. The effectiveness of randomization with reconstruction for categorical attributes is exemplified. 
Wang et al. (2010) proposed a modified algorithm called PPFDM and related computation technique based on the Frequent Data Mining (FDM) to preserve privacy. The process involved the computation of total support count along with the privacy-preserved technique while ensuring the local large item-set and local support count source is covered. Thus, the time needed for the communication is saved and secured the distributed data privacy at each site. The experimental results demonstrated the effectiveness and suitability of the method for practical application, especially in privacy preservation during mining process.

Nguyen et al. (2012) presented an Enhanced M. Hussein et al's Scheme (EMHS) for secured privacy association rules mining, where horizontally distributed database is used. EMHS (developed in 2008) is capable to modify the privacy and efficiency with increasing number of sites. The efficiency of EMHS is discerned to be much better than MHS, particularly for databases with increasing number of sites. A second approach is also presented for the other types of datasets. It is important to solving the collusion of Initiator and Combiner. Om Kumar et al. (2013) used WEKA to predict the patterns in a single cloud. By using cloud data distributor with a secured distributed approach they provided an effective solution that prevented such mining attacks on cloud. Thus, it made the cloud a secured platform for service and storage.

Mokeddem and Belbachir (2010) proposed a distributed model to perform classassociation rules detection for shared-nothing framework. The solution of the proposed model is one of the fastest known sequential algorithms (FP-growth) which is extended to produce classification rules in a parallel setting. By using the proposed system, the data replication is avoided on these sites with an option to communicate the required information. These choices are evaluated by performing experimentations, which permitted us to analyze several important aspects such as accuracy, scalability, speedup, memory usage, communication, synchronization, and also the load balancing. Ibrahim et al. (2012) developed a practical cryptographic model to calculate the KNN categorization over the distributed cloud databases. Their experiments demonstrated similar accuracy of the proposed as the naive scheme without security. It is believed that such schemes may mitigate the users concerns and accelerate the paces towards the high adoption of cloud computing. The extension of our secure classifier to work in the malicious adversary security model will be reported elsewhere.

Patel et al. (2012) proposed an operative algorithm to protect the secrecy distributed over K-Means cluster using Shamir's secret sharing model. The proposed approach computed the cluster mean collaboratively and prevented the role of trusted third party. Upon comparison, it is observed that the proposed framework is orders of magnitude faster as compared to oblivious polynomial evaluation and homomorphic encryption techniques in terms of computation cost and more reliable for huge databases. It is essential to extend the proposed algorithm in vertical partitioning in the presence of malicious adversary model. In addition, the results from a realistic distributed emulation are worth looking. Kumbhar and Kharat (2012) analysed and compared different techniques used for Privacy Preserving Association Rule Mining (PPARM). The algorithm based on cryptography techniques, Homomorphic encryption, Secure Scalar product and Shamir's secret sharing technique are employed to satisfy the privacy constraints for vertically partitioned dataset. However, for horizontally partitioned dataset 
the algorithm with the combination of RSA public key cryptosystem and Homomorphic encryption scheme are used. Paillier cryptosystem is employed to determine the global supports. In practice, while calculating c.count collaboratively, participant may deviate from algorithm and lead malicious behaviour. But algorithm is semantically secured and prevents collusive behaviour with accurate results.

Nix et al. (2012) implemented two sketching protocols for the scalar (dot) product of two vectors which are used as sub-protocols in larger data mining tasks. Results through extensive experimentations revealed their high accuracy, low data leakage, and orders of magnitude improved efficiency. The security properties of these approximations under a security definition are also analyzed. In contrast to the previous definitions these are found to be very efficient approximation protocols. It is worth to explore the use of these dot product protocols in other data mining tasks such as support vector machines, neural networks, and clustering. The notion of a secure approximation and determination of the relaxation extent of the posed restrictions by the security model need to be looked at.

Keshavamurthy et al. (2013) demonstrated that GA approach possessed two potential advantages than traditional frequent pattern mining algorithm. It is found that in frequent pattern mining, the population is formed only once. Conversely, in GA method the population is formed for each generation that maximizes the sample set. However, the major drawback of GA approach is connected to the duplication in its sequential generations. For privacy preservation data mining over distributed dataset, the key goal is to permit computation of collective statistics for complete database with assurance of the privacy for confidential data of the contributing databases. Hence, the algorithms for privacy preservation needs further improvement based on the trade-offs between reconstruction accuracy and privacy. On top, the fitness function of GA plays an important role and the convergence of search space is directly proportional to the effectiveness of fitness function. In other words, superior fitness functions for a given problem leads to faster convergence of GA.

\section{K-anonymity based PPDM}

For the sake of clarity, it is customary to render two important definition of K-anonymity.

The first definition tells that: $Q I$ being a quasi-identifier for a given table $U$ with $T\left(A_{1} \ldots A_{n}\right), \mathrm{f}_{C}: U \rightarrow T, f_{g}: T \rightarrow U^{\prime}$, where $U \subseteq U^{\prime}$, a quasi-identifier of $T\left(Q_{T}\right)$ is a set of attributes $\left\{A i \ldots A_{J}\right\} \subseteq\left\{\mathrm{A}_{1} \ldots \mathrm{A}_{n}\right\}$, where $\exists p_{i} \in U$ such that $f_{g}\left(f_{c}\left(p_{i}\right)\left[Q_{T}\right]\right)=p_{i}$ (Sweeney 2002). The second definition is stated as follows: a table $T$ satisfies $K$-anonymity if for every tuple $\mathrm{t} \in \mathrm{T}$ there exist $k-1$ other tuples $t_{i 1} t_{i 2} \ldots t_{k-1} \in T$ such that $t_{i 1}[\mathrm{C}]=t_{i 2}[\mathrm{C}]=\ldots t_{i k-1}[\mathrm{C}]$ for all $\mathrm{C} \in \mathrm{Q}_{\mathrm{T}}$ (Machanavajihala et al. 2007).

A scalable solution for each repetition can examine at least one generalization for each attribute involved in the linking. (Wang et al. 2004) studied the data mining as a approach used for data masking called data mining based on privacy protection. The data mining methods are inspected in terms of data generalization concept, where the data mining is performed by hiding the original information instead of trends and patterns. After data masking, the common data mining methods are employed without any modification. Two key factors, quality and scalability are specifically focused. The quality issue is settled via the trade-off between privacy and information. The scalability issue 
is established employing new data architecture while focusing on good generalizations. Loukides and Gkoulalas-divanis (2012) proposed a novel technique to anonymize the data by satisfying the data publishers' utilization necessities experiencing low information loss. An accurate information loss measure and an effective anonymization algorithm are introduced to minimize the information losses. Experimental investigations on click-stream and medical data revealed that that the proposed technique allowed more reliable query answers than the state state-of-the-art techniques which are equivalent in terms of efficiency. This work opens up several promising avenues for future research. These include examining how UAR can be extended to guard against both identity and sensitive information disclosure and how to produce anonymized data with guaranteed utility in certain data mining tasks, such as classification and association rule mining. Friedman et al. (2008) extended the definitions of K-anonymity to prove that the data mining model does not violate the K-anonymity of the clients represented in the learning examples. A tool is provided to determine the amount of anonymity retained during data mining. The proposed approach showed its employment capability to different data mining problems including classification, association rule mining and clustering.

The K-anonymity is further combined with data mining approach to protect the respondent's identity. Ciriani et al. (2008) highlighted the potential threats to K-anonymity, which are raised via the implementation of mining to collect data and analyses of two main techniques to join K- anonymity in data mining. The different approaches employed to detect K-anonymity violations are also described. Subsequently, the elimination of these approaches in association rule mining and classification mining are emphasized. He et al. (2011) proposed an algorithm based on clustering to produce a utility-friendly anonymized version of micro data. This method is found to outperform the non-homogeneous technique where the size of QI-attribute is greater than 3 . They achieved a clustering-based K-anonymity algorithm, which revealed considerable improvement in the utility performance when applied to several real datasets. Recently, K-anonymous privacy preservation is widely employed. Further modification appeared to be increasingly difficult without resolving several issues. Patil and Patankar (2013) examined the standard K-anonymity techniques and its applications. Some of the multidimensional K-anonymous investigation is carried out. Yet, the present are multidimensional data sets based K-anonymity algorithms using nearest neighbour strategy are useful to enhancing the quality of anonymity and reducing the information loss.

Lately, K-anonymity became one of the most important topics for privacy preservation. This can effectively avoid privacy leaks due to link attacks. Certainly, K-anonymity is one of the widely used approach in all fields (Zhu and Chen 2012). Soodejani et al. (2012) employed a version of the chase termed as standard chase, which put some restrictions on the dependencies and constrains, such as being positive and conjunctive. This area is prospective for future study in fathering investigations on the applicability of other versions of the chase in the method. The anonymity principle of their method reveals some similarities to the L-diversity privacy model. Investigation of other privacy models such as t-closeness may provide a stronger privacy model for the proposed method with extreme usefulness. Karim et al. (2012) proposed a numerical method to mine maximal frequent patterns with privacy preserving capability. This method showed an efficient data transformation technique, a novel encoded and compressed lattice 
structure and MFPM algorithm. The proposed lattice structure and MFPM algorithm reduced both the search space as well as the searching time. The experimental results displayed that the MFPM algorithm outperformed PC_Miner and existing maximal frequent pattern mining algorithms. Besides the lattice structure, it outperformed FP-like tree and PC_tree algorithm as well.

Loukides et al. (2012) proposed a rule-based privacy model that allowed data publishers to express fine-grained protection requirements for both identity and sensitive information disclosure. Based on this model, they developed two anonymization algorithms. Their first algorithm worked in a top-down fashion, employing an efficient strategy to recursively generalize data with low information loss. Conversely, the second algorithm used sampling and a mixture of bottom-up and top-down generalized heuristics. This greatly improved the scalability and maintained low information loss. Extensive experimentations show that these algorithms significantly outperformed the state-of-the-art in context of recalling data utilization, while keeping good protection and scalability. It provides a foundation for some future studies. First, while identity and sensitive information disclosure are the main concerns in data publishing, it is worth examining membership disclosure, in which inferring whether an individual's record is contained in the published data is to be prevented. Second, it is worth to extend the proposed approach to anonymize disk-resident data with small memory consumption and I/O overhead.

Vijayarani et al. (2010b) studied K-anonymity as an interesting approach to protect micro data related to public or semi-public sectors from linking attacks. The possible threats to K-anonymity approach is described in detail. Particularly, the problems related to data and the approaches are identified to combine K-anonymity in data mining. Nergiz et al. (2009) improved and extended the definitions of K-anonymity to manifold relations definitions of K-anonymity expression. It is shown that earlier developed techniques either failed to secure privacy or as a whole reduced the data utilization, and data protection in a multiple relations setting. A new clustering algorithms is introduced to obtain multi-relational anonymity. Experimental results illustrated that the proposed technique is an effective approach in terms of utility and efficiency. Support for arbitrary schemes with multiple private entities must be considered.

The problem of secured outsourcing of frequent itemset mining on the multi-cloud environments is studied by Tai et al. (2013). Concerning the challenges in big data analysis, they suggested to partition the data into several parts and outsourced each part independently to different cloud based on pseudo-taxonomy, anonymization technique, known as KAT. They proposed DKNT to ensure the privacy security for each partial data outsourced to different clouds. Experimental results demonstrated excellent achievement in terms of protection and better computation efficiency as compared to those on a single machine. Tai et al. (2010) presented K-support anonymity, which provided protection against a knowledgeable attacker with the exact support information. To achieve the K-support anonymity, a pseudo taxonomy tree is introduced with the third party mine for the generalized frequent item-sets. The construction of the pseudo taxonomy tree facilitated the hiding of the original items and limited the fake items introduced in the encrypted database. The results showed very good privacy protection with moderate storage overhead. K-anonymity is further enhanced and improved by Pan et al. (2012). They analyzed and compared the developed K-anonymity models and discussed their 
applications. The modified K-anonymity models such as the L-diversity, $(\alpha, \mathrm{K})$-anonymity and $(\alpha, \mathrm{L})$-diversification $\mathrm{K}$-anonymity overcome the existing limitations related to privacy. Few K-anonymous methods are employed in obtaining the main technology.

Based on suppression, Deivanai et al. proposed a new K-anonymity technique called 'kactus' (Deivanai et al. 2011). In the proposed technique, multi-dimensional suppression is performed. The values are suppressed to a certain records based on other attributes without using the domain hierarchy trees. Thus, this approach identified the attributes independent of classification of the data records and suppressed these values to comply with K-anonymity. This approach is implemented on different database to determine its accuracy and efficiency and compared with other K-anonymity based techniques. It is affirmed that in a multiparty environment, the anonymization can be performed with perturbation to preserve privacy. A new definition of K-anonymity model for effective privacy protection of personal sequential data is introduced (Monreale et al. 2014). This method transformed the sequential datasets into a K-anonymous form, while preserving the utility of data with reference to a variety of analytical properties. A series of experimentation on different real-life sequential data bases exhibited that the proposed approach substantially secured the sequential pattern mining results not only in terms of extracted patterns but also the support. Furthermore, the results appeared extremely interesting in the case of dense datasets.

Nergiz and Gök (2014) and Nergiz et al. (2013) introduced the hybrid generalizations. It not only performed the generalizations, but also involved the mechanism for data relocation. In data process, the position of certain cells is changed to some populated indistinguishable data cells. The relocation process helped to generate anonymizations of finer granularity and ensured underlying privacy. The data relocation is a trade-off among the utilization and reliability of the data, where the trade-off is controlled by the provider parameter. The results revealed that a small number of relocations could enhance the utility as compared to the heuristic metrics and query answering accuracy. A Hybrid generalizations mechanism to relocate the data is introduced (Nergiz and Gök 2014). In data relocation process, data cells are relocated to certain populated small groups of tuples which remained distinguishable from each other. Again, the data relocation helped to generate anonymizations of finer granularity which ensured the data privacy. It is demonstrated that a small number of relocations could remarkably enhance the utility. New hybrid algorithms can be designed for other privacy metric such as diversity, $(\alpha, \mathrm{k})$-anonymity or $\delta$-presence. This would be crucial in addressing different types of adversaries. There is also room for improvement of the proposed hybrid algorithms. For example, one can design hybrid algorithms that would theoretically bound to the probability of identification against algorithm-aware adversaries.

Zhang et al. (2013a, 2014a) investigated the issues related to scalability of sub-tree anonymization for huge data storage on cloud. They developed a hybrid approach along with Top-Down Specialization (TDS) and Bottom-Up Generalization (BUG) techniques. In this method, one of the two components is selected automatically by comparing K-anonymity parameter with workload balancing point which is defined by the clients. Both TDS and BUG are obtained in a scalable way via a series of deliberately designed Map Reduce jobs. Based on the contributions herein, it is worth exploring the next step on scalable privacy preservation aware analysis and scheduling on large-scale 
datasets. Later, Zhang et al. (2014b) introduced a two-phase TDS technique based on Map Reduce on cloud. In the first phase, the data sets are anonymized and partitioned in parallel and intermediate results are generated. In the second phase, these intermediate results are aggregated for further anonymization to produce consistent $\mathrm{K}$-anonymous datasets. The Map Reduce on cloud is employed for data anonymization and a group of data is designed deliberately to concretely achieve the specific computation in a scalable way. The results from the implementation of this method on real-world datasets displayed that the presence of scalability and competence of TDS made the performance much better than existing methods. They have presented an efficient quasi-identifier index based technique to preserve the privacy over incremental datasets on cloud. In the proposed technique, QI-groups (QI: quasi-identifier) are listed using domain values in the current generalization level, which allowed the access only to a small portion of records in any database rather than admittance to the whole data base (Zhang et al. 2013b, c). In addition, Ding et al. 2013) introduced a distributed anonymization protocol for privacy-preserving data publishing from multiple data providers in a cloud system. Their method performed a personalized anonymization to satisfy every data provider's requirements and the union formed a global anonymization to be published. They also presented a new anonymization algorithm using R-tree index structure.

\section{Shortcomings of PPDM methods}

Currently, several data mining techniques are available to protect the privacy. Broadly, the privacy preserving techniques are classified according to data distribution, data distortion, data mining algorithms, anonymization, data or rules hiding, and privacy protection. Table 1 summarizes different techniques applied to secure data mining privacy. Intensive research findings over the decades revealed that the existing privacy preserving data mining search approaches are still suffer from major incompleteness including the distributed clients' data to multi semi honest providers, the overhead of computing global mining, incremental data privacy issue in cloud computing, integrity of mining result, utility of data, scalability and overhead performance. Thus, a strong, efficient, and scalable model is essential to surmount these shortcomings. Furthermore, proper anonymization of data is needed to protect the privacy of each client prior to publish. The connection between personal data and personal identification should be vanished.

Table 1 Description of PPDM methods

\begin{tabular}{ll}
\hline PPDM methods & Description \\
\hline $\begin{array}{l}\text { Data distribution } \\
\text { Data distortion }\end{array}$ & $\begin{array}{l}\text { May contain vertically or horizontally partitioned data } \\
\text { Contain perturbation, blocking, aggregation or merging, swapping and sampling }\end{array}$ \\
$\begin{array}{ll}\text { Data mining algorithms } \\
\text { Encloses classification mining, association rule mining, clustering, and Bayesian net- } \\
\text { Works etc }\end{array}$ \\
$\begin{array}{l}\text { K-anonymity } \\
\text { L-diverse }\end{array}$ & $\begin{array}{l}\text { Achieve the anonymization } \\
\text { Taxonomy Tree }\end{array}$ \\
Randomization & Attributes the generalization to limit the information leakage \\
Privacy protection & An un-sophisticated and valuable technique to hide the individual data in PPDM \\
\end{tabular}


Such an anonymization must not only satisfy underlying privacy requirements but also safeguard the utility of the data.

Undoubtedly, K-anonymity is an effective method of privacy protection in data mining. However, several demonstrated that the data processed by this method often failed to overcome some attacks and are susceptible to internet phishing. Consequently, the future privacy preserving data mining based K-anonymity needs an advance data infrastructure to support the combination of present data functionality. This would definitely fulfil the requirements of different kinds of clients and communities. Although the present search algorithms are able to speed up the retrieval process, but they do not scale up to large volume of data because of the linear increase of response time with the amount of the searched datasets. The proposed techniques for the searching of distributed large data among many cloud providers must possess the ability to preserve privacy, must be scalable, efficient, compatible and good for utility as well as integrity. Table 2 enlists some relevant studies on privacy preserving data mining as well as their notable merits and de-merits. Table 3 outline the categorization of current studies.

\section{Conclusion}

An inclusive overview on PPDM techniques based on distortion, associative classification, randomization, distribution, and k-anonymization is presented. It is established that PPDM is appeared progressively common due to easy sharing of privacy sensitive data for analysis. The notable advantages and obvious disadvantages of current studies are emphasized. Presently, Big Data are often shared across sectors such as health, military and others, and transverses across Business-to-Businesses, Entities-to-Entities and Government-to-Government. Thus, the preservation of privacy against disclosure and attacks are of critical concern. Several big organizations and governments worldwide being totally dependent on information communications via internet expressed grave concerns over privacy issues. Consequently, the rapid development of IT faced new challenges to PPDM. Data mining possesses being the capability to extract and mine vast sea of interesting patterns or knowledge from a huge amount of data requires absolute security. The main idea of PPDM is to incorporate the traditional data mining techniques in transforming the data to mask sensitive information. The major challenge is to efficiently transform the data and recover its mining outcome from the transformed one. Furthermore, the incompleteness of previous studies indicated forced us to engage in an extensive inspection of the problems of distributed and published data for sharing and mining. Consequently, the overhead for global mining computing, preserving privacy of growing data, the integrity of mining result, the utility of data, the scalability and overhead performance in the context of PPDM are examined. There is an urgent necessity to develop a strong, efficient, and scalable model to surmount these issues. In this regard, we identified the gaps and weaknesses of existing literatures and analyzed them for further significant enhancements, more robust privacy protection, and preservation. This exhaustive and informative review article is hoped to serve as taxonomy for navigating and comprehending the research advancements towards PPDM. 


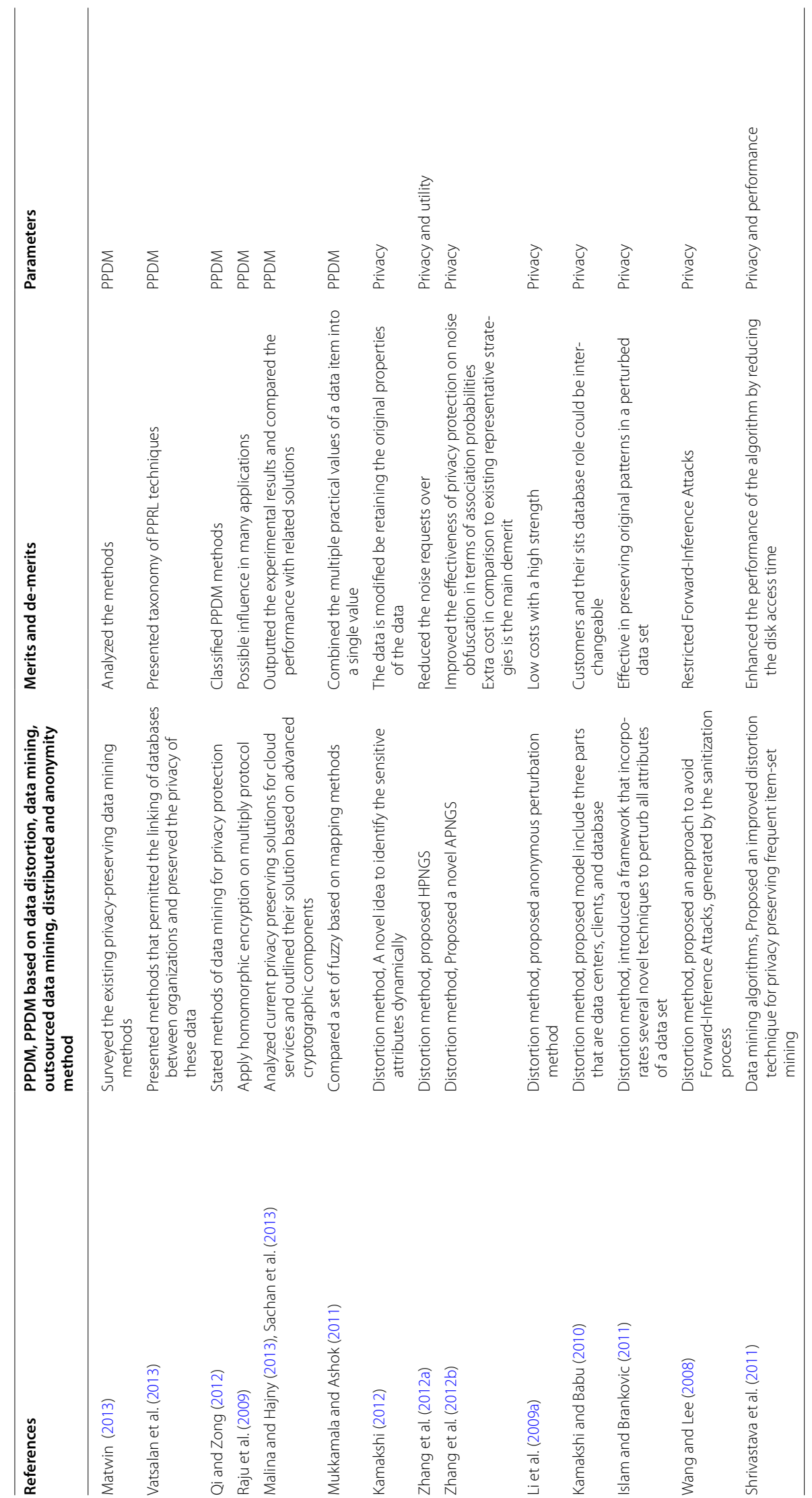




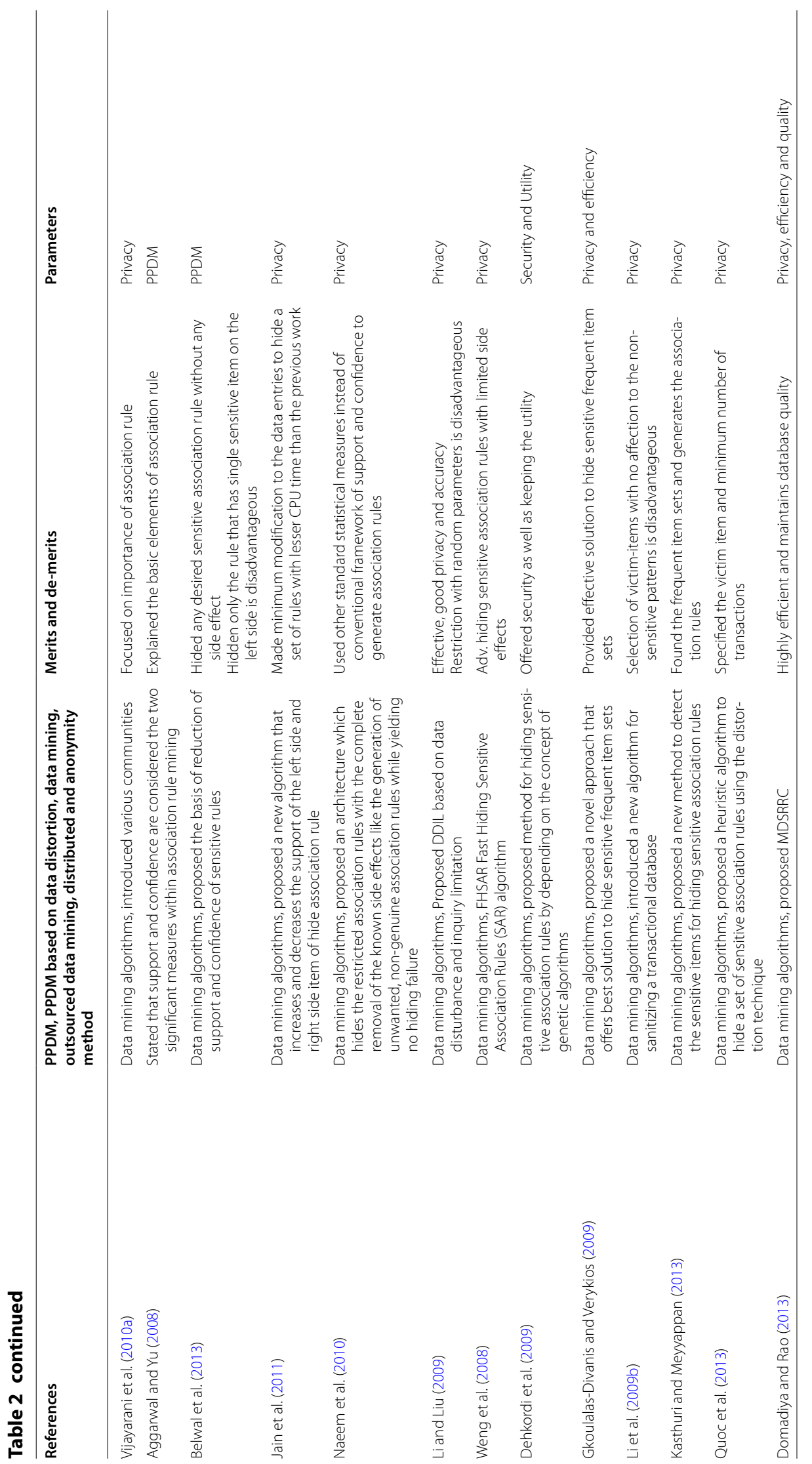




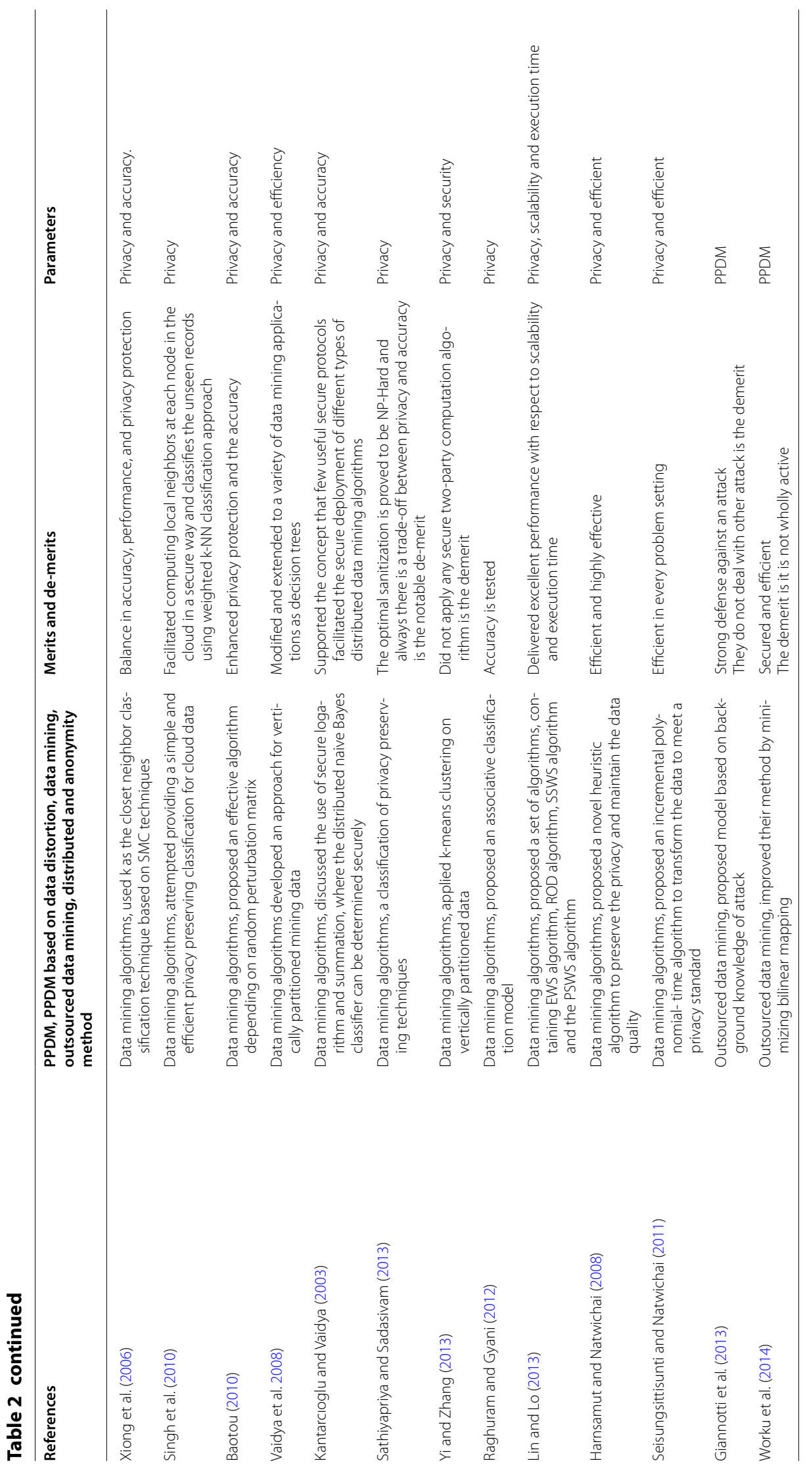




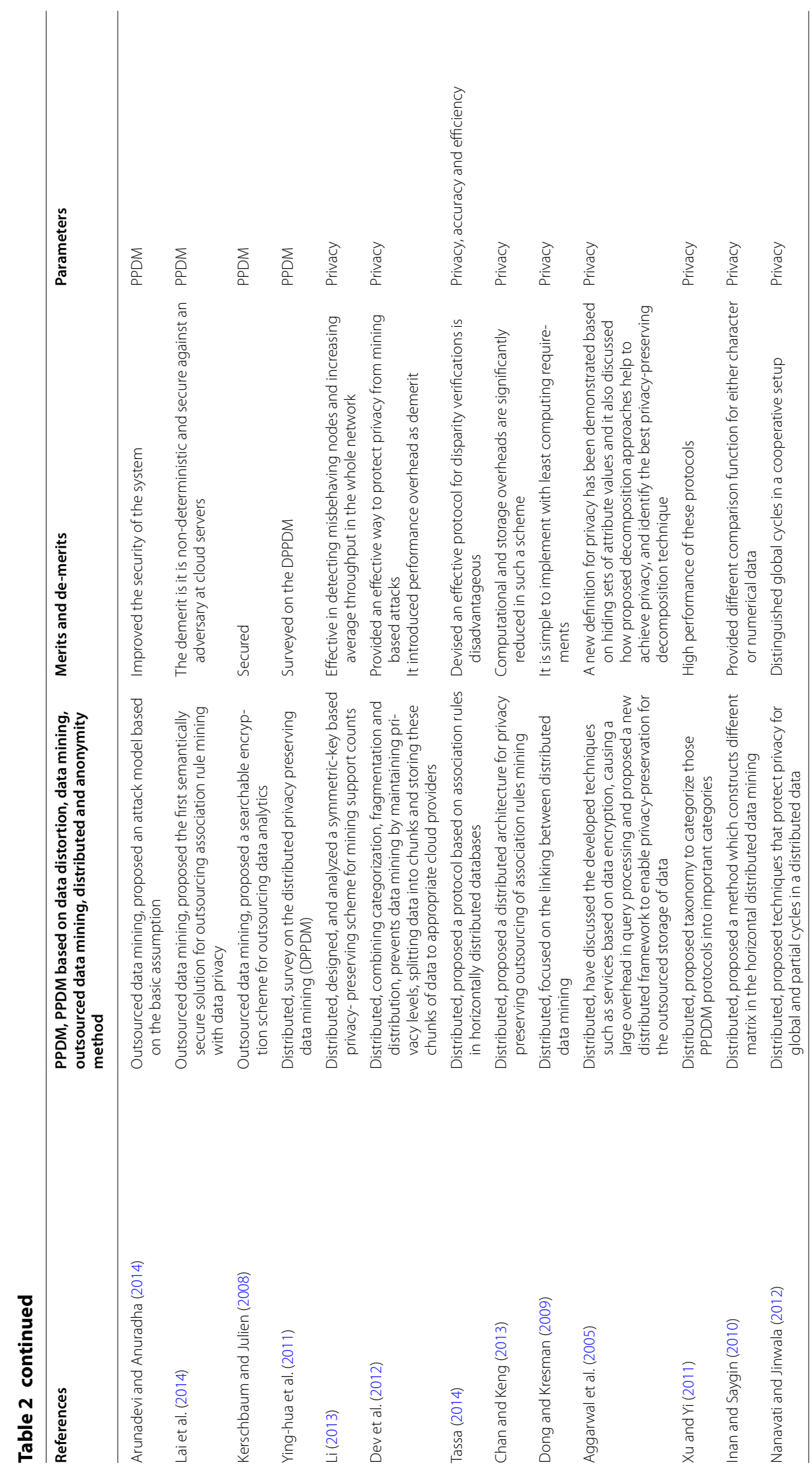




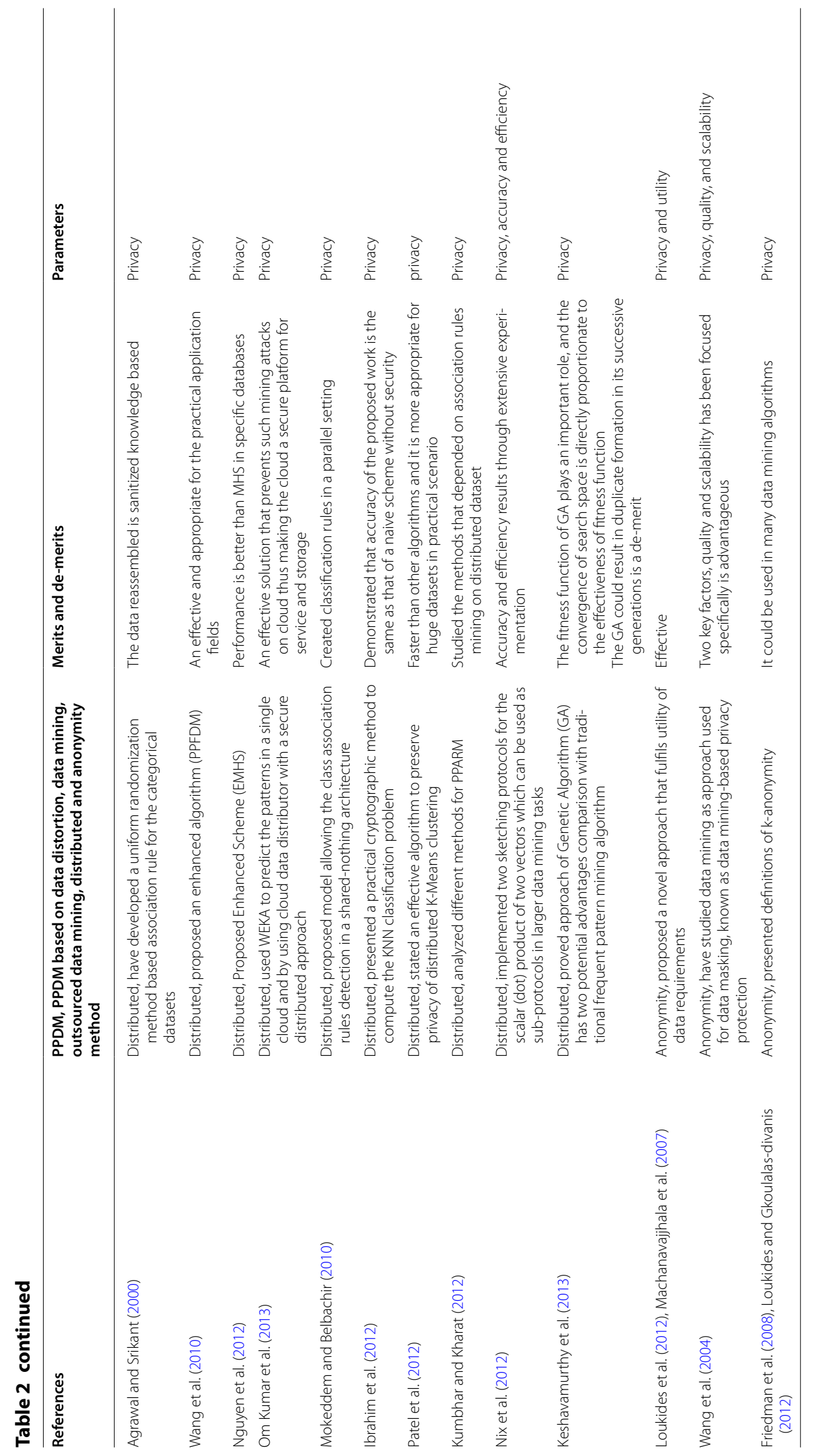




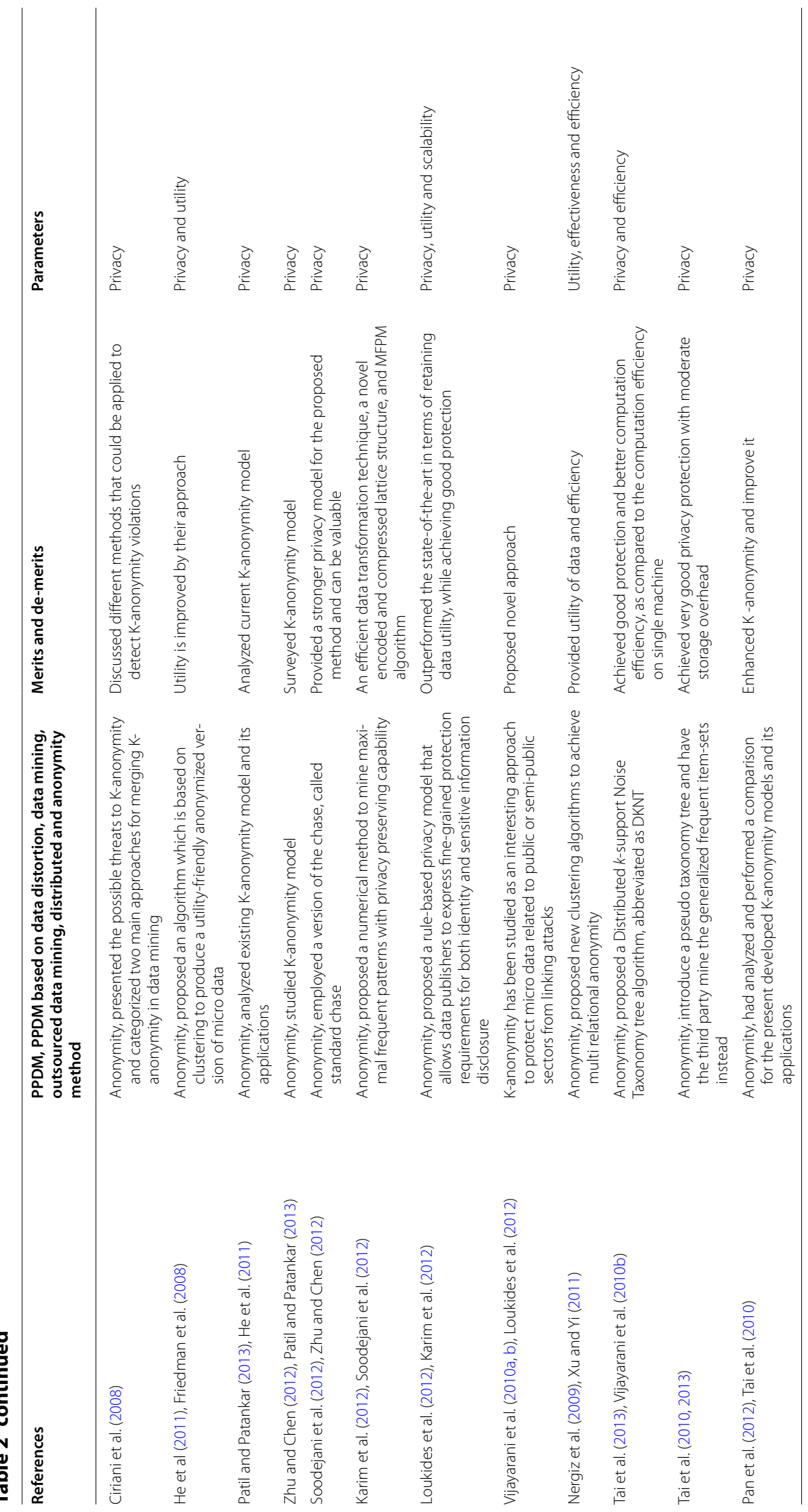




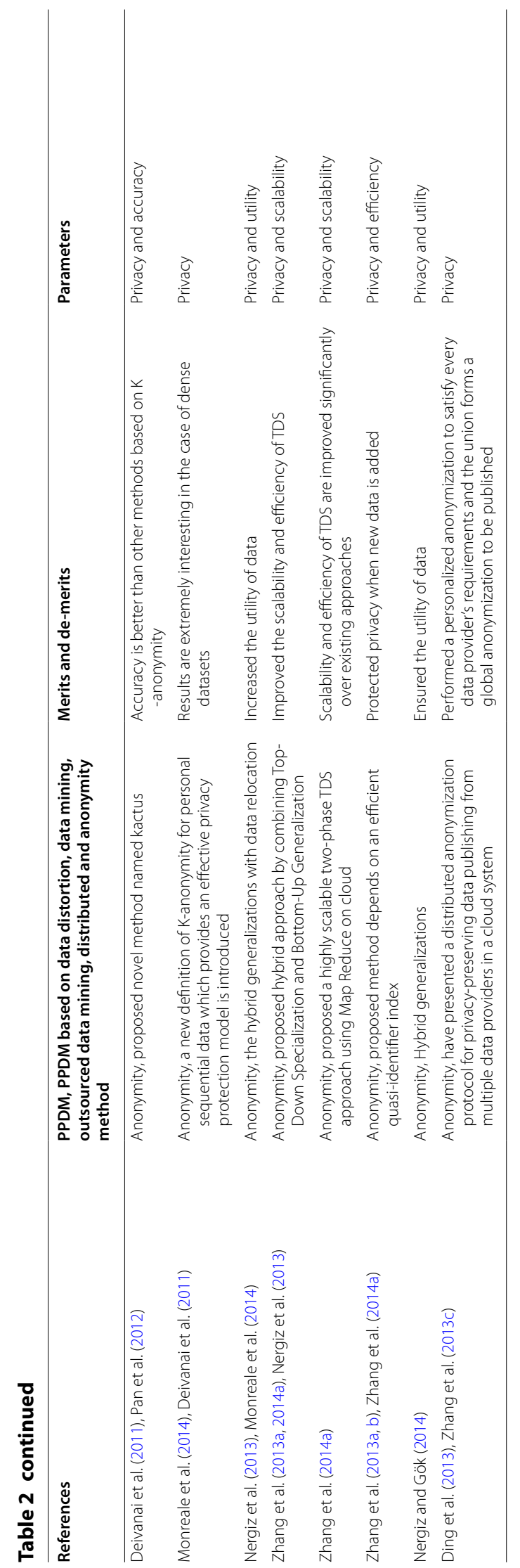




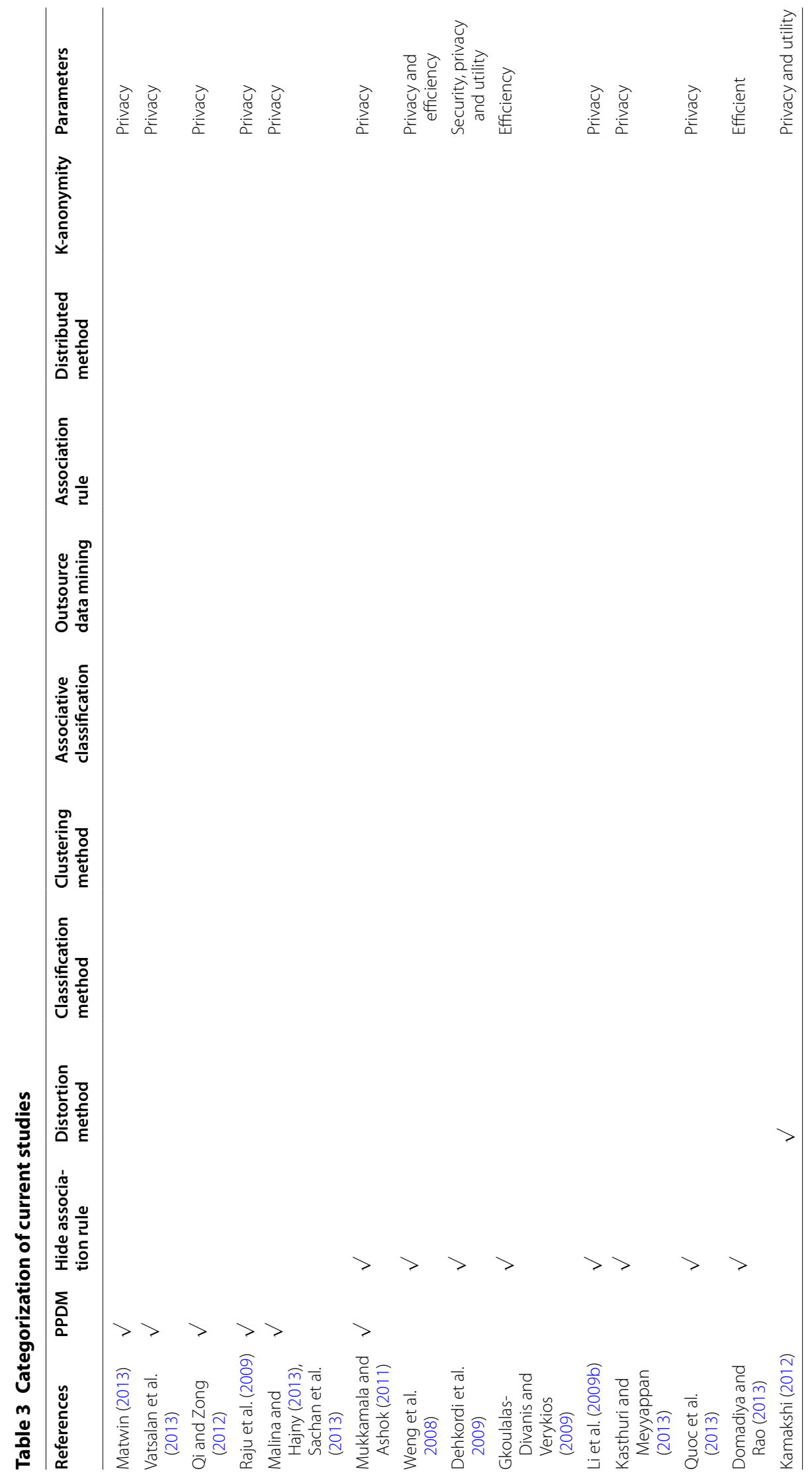




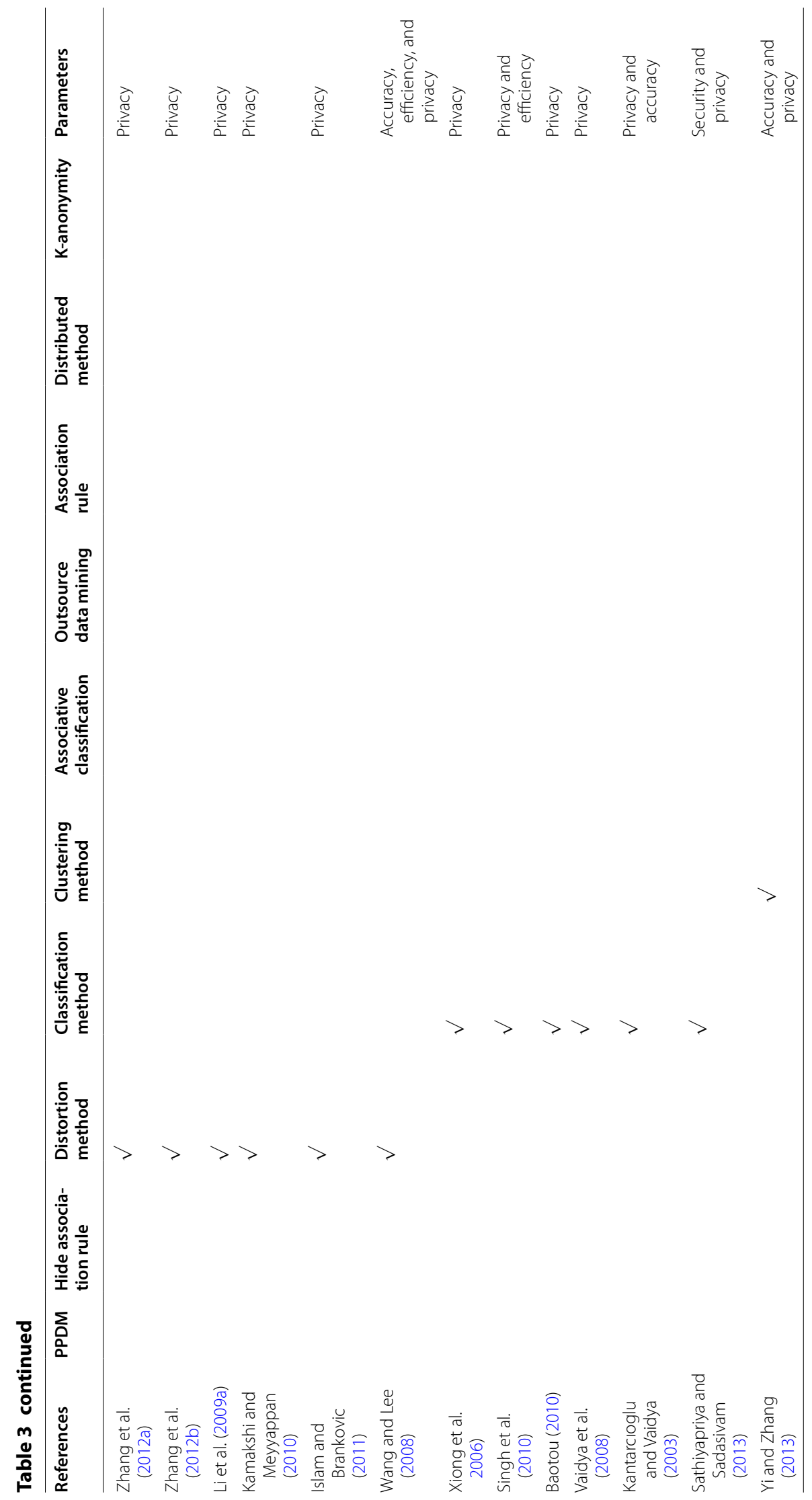




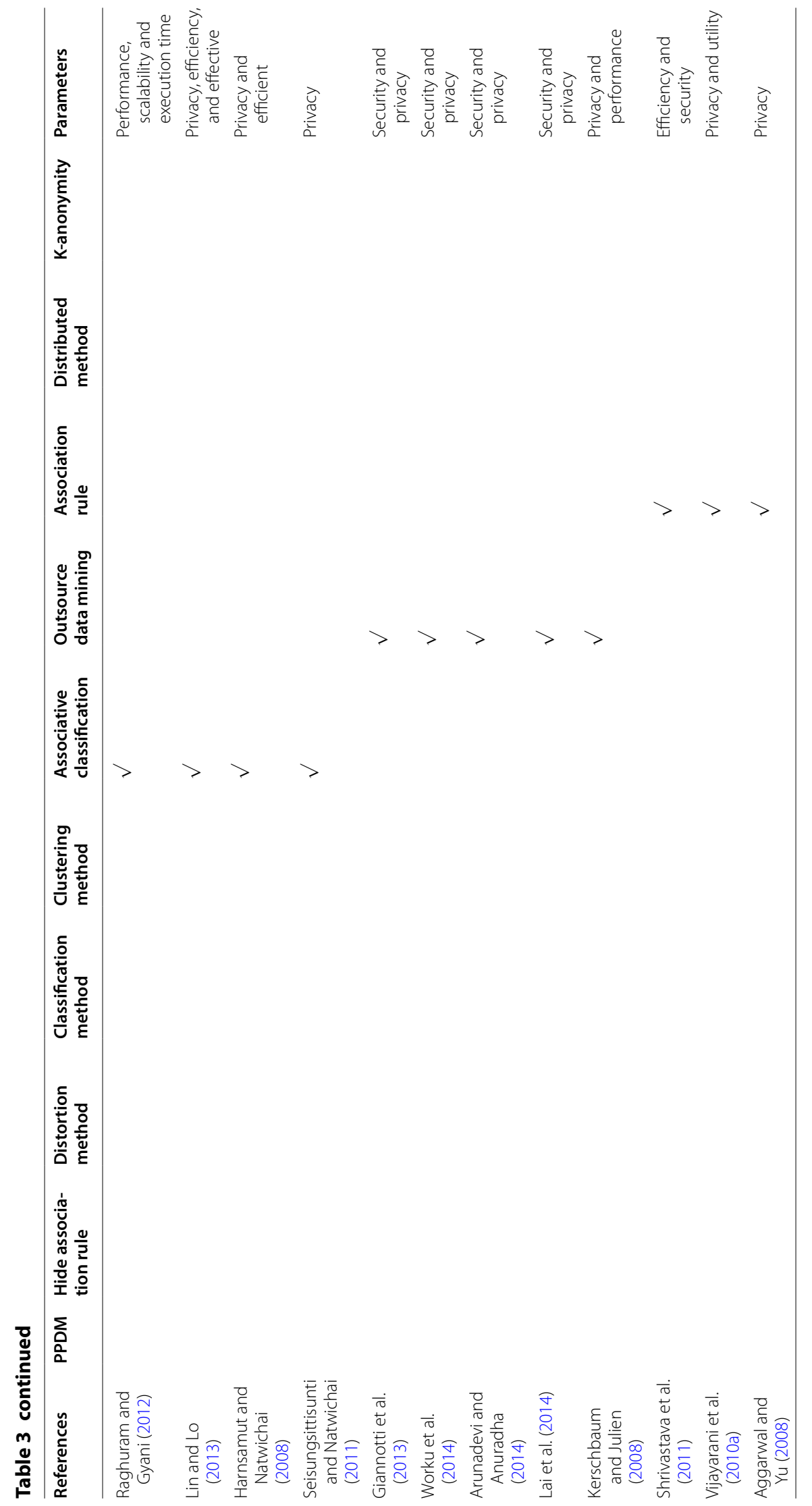




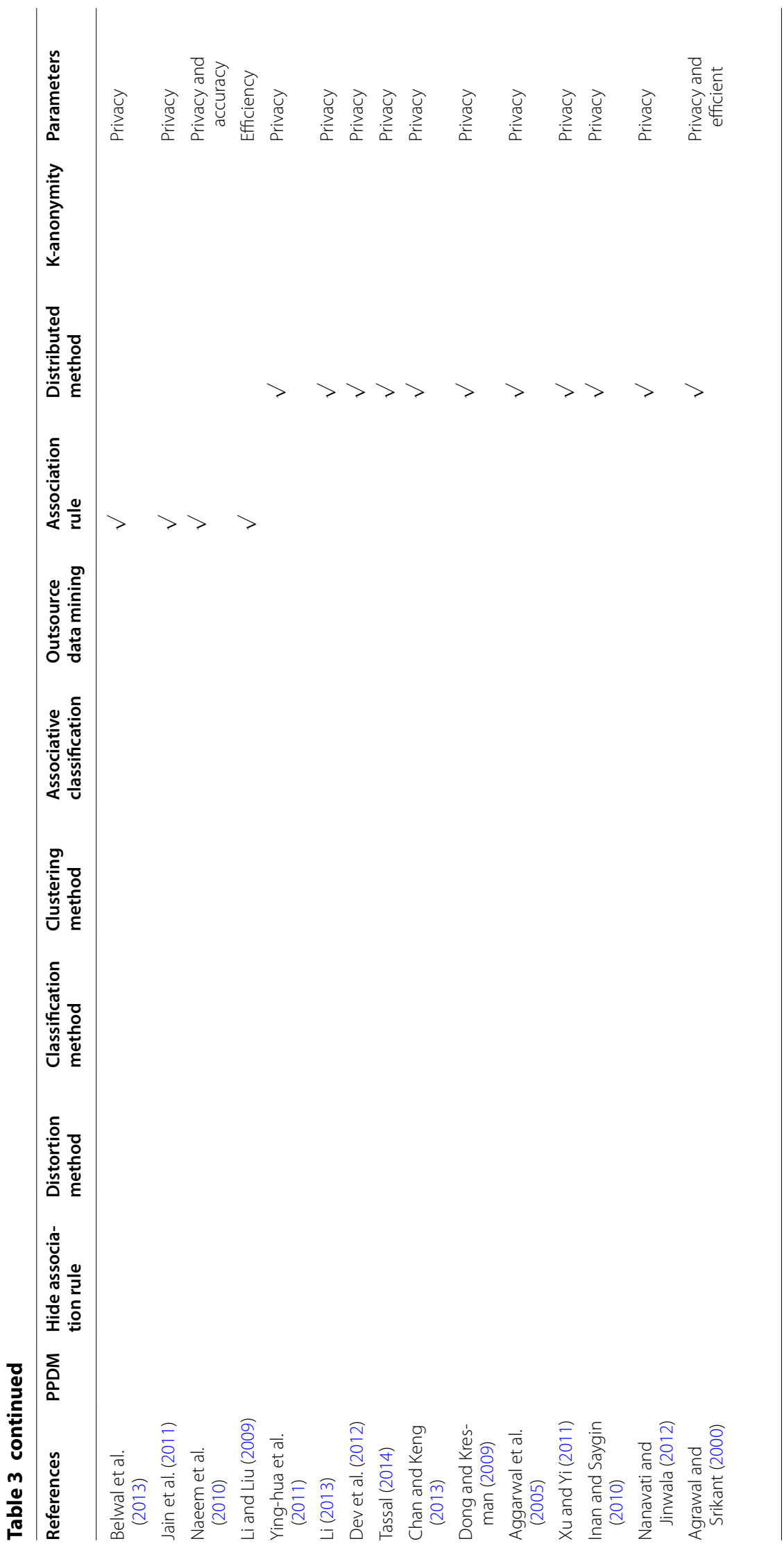




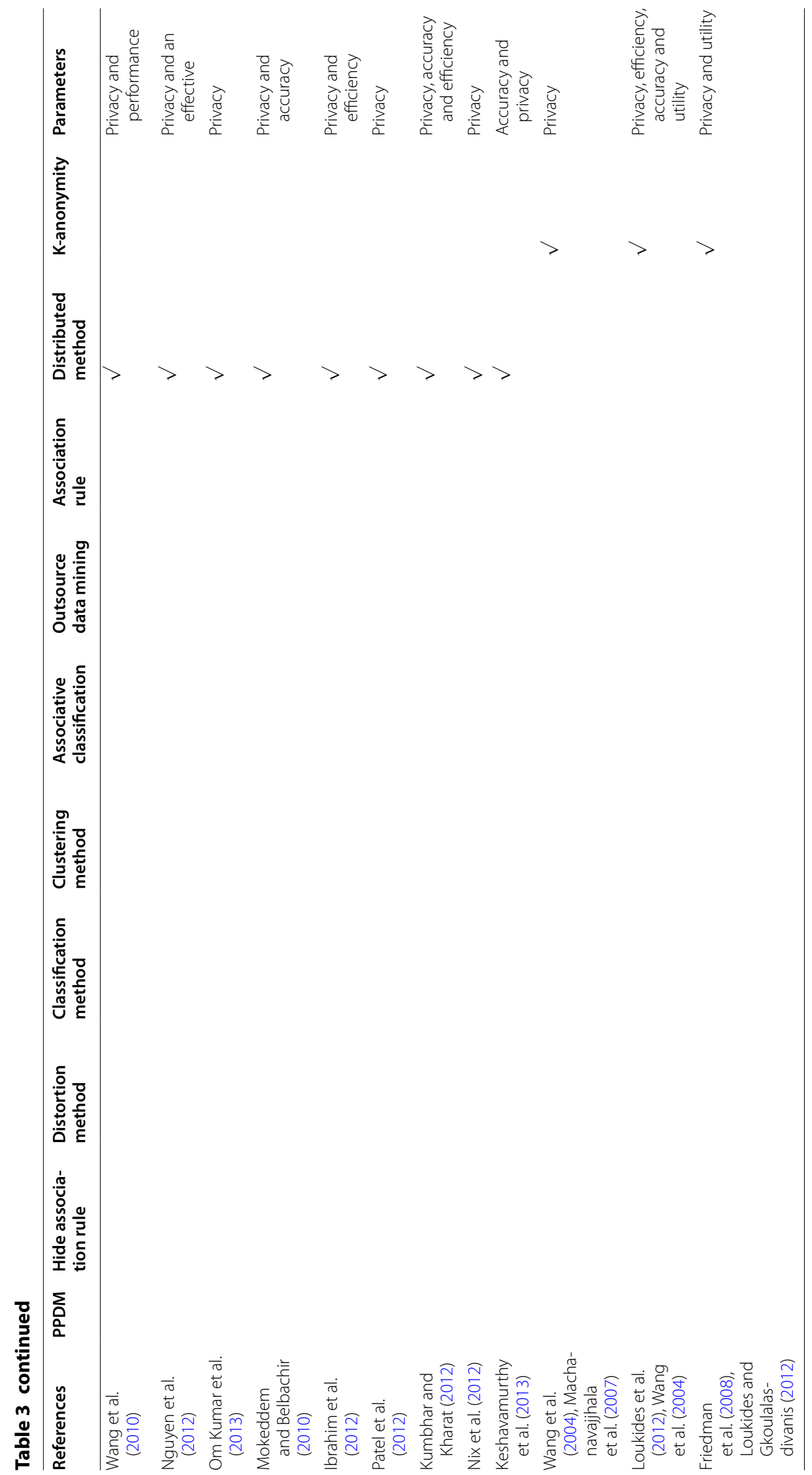




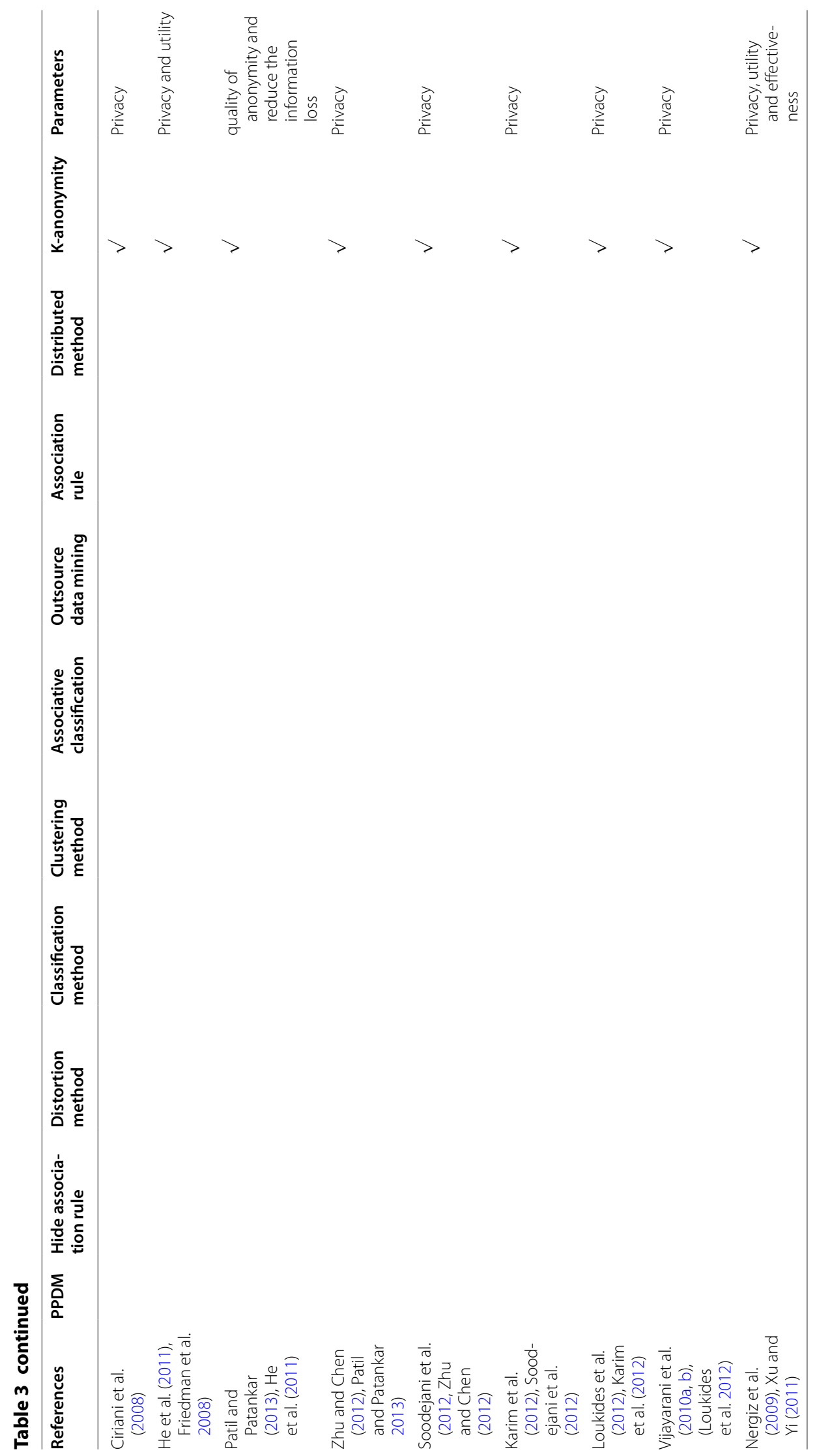




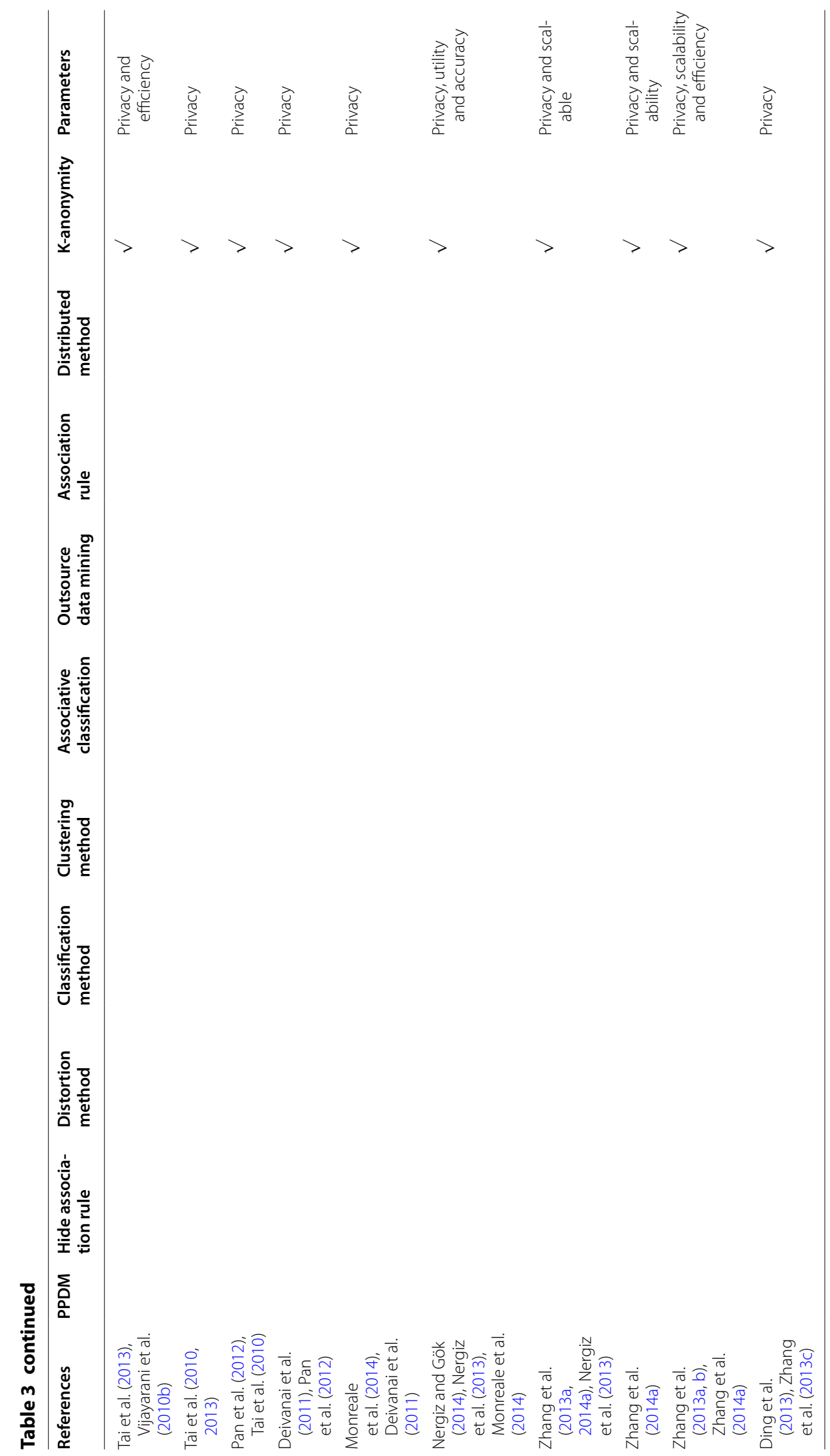




\section{Authors' contributions}

YAASA (phd student) carried out the PPDM studies, made substantial contributions to the concepts and the design of this article. Furthermore, she collected the data on PPDM, analyzed and interpreted the data as well as participated in the sequence alignment to articulate the manuscript. MS (Associate Prof. Dr.) designed and drafted the article, made critical revision for important intellectual content with thorough supervision. MAR (Dr.) aligned the article sequentially, designed, coordinated and helped to draft the manuscript. All authors read and approved the final manuscript.

\section{Author details}

${ }^{1}$ Faculty of Computing, University Technology Malaysia, UTM, 81310 UTM Skudai, Johor, Malaysia. ${ }^{2}$ Department of Computer Science, College of Education, Ibn Rushd, Baghdad University, Baghdad, Iraq.

\section{Acknowledgements}

Authors are thankful to UTM library for providing several useful references.

\section{Competing interests}

The authors declare that they have no competing interests.

Received: 2 March 2015 Accepted: 28 October 2015

Published online: 12 November 2015

\section{References}

Aggarwal G, Bawa M, Ganesan P (2005) Two can keep a secret: a distributed architecture for secure database services. CIDR

Agrawal R, Srikant R (2000) Privacy-preserving data mining. In: Proceedings of the 2000 ACM SIGMOD international conference on management of data_SIGMOD '00, vol 29, no 2. pp 439-450. http://doi.org/10.1145/342009.335438

Aggarwal CC, Yu PS (2008) A general survey of privacy-preserving data mining models and algorithms. In: Privacy preserving data mining, Chap 2. Springer, New York, pp 11-52. http://doi.org/10.1007/978-0-387-48533

Arunadevi M, Anuradha R (2014) Privacy preserving outsourcing for frequent itemset mining. Int J Innov Res Comp Commun Eng 2(1):3867-3873

Baotou T (2010) Research on privacy preserving classification data mining based on random perturbation Xiaolin Zhang Hongjing Bi. 1-6

Belwal R, Varshney J, Khan S (2013) Hiding sensitive association rules efficiently by introducing new variable hiding counter. In: IEEE international conference on service operations and logistics, and informatics, 2008, IEEE/SOLI 2008, vol 1, pp 130-134. doi:10.1109/SOLI.2008.4686377

Chan J, Keng J (2013) Privacy protection in outsourced association rule mining using distributed servers and its privacy notions, pp 1-5

Ciriani V, Vimercati SDC, Foresti S, Samarati P (2008) k-anonymous data mining: a survey. In: Privacy-preserving data mining. Springer, New York, pp 105-136

Dehkordi MNM, Badie K, Zadeh AKA (2009) A novel method for privacy preserving in association rule mining based on genetic algorithms. J Softw 4(6):555-562. http://doi.org/10.4304/jsw.4.6.555-562

Deivanai P, Nayahi J, Kavitha V (2011) A hybrid data anonymization integrated with suppression for preserving privacy in mining multi party data. In: IEEE international conference on recent trends in information technology (ICRTIT)

Dev H, Sen T, Basak M, Ali ME (2012) An approach to protect the privacy of cloud data from data mining based attacks. In: IEEE 2012 SC companion high performance computing, networking, storage and analysis (SCC)

Ding X, Yu Q, Li J, Liu J, Jin H (2013) Distributed anonymization for multiple data providers in a cloud system. In: Database systems for advanced applications. Springer, Berlin, Heidelberg

Domadiya NH, Rao UP (2013) Hiding sensitive association rules to maintain privacy and data quality in database. In: IEEE 3rd international on advance computing conference (IACC), pp 1306-1310

Dong R, Kresman R (2009) Indirect disclosures in data mining. In: Fourth international conference on frontier of computer science and technology, FCST'09

Friedman A, Wolff R, Schuster A (2008) Providing k-anonymity in data mining. VLDB J 17(4):789-804

Giannotti F, Lakshmanan LVS, Monreale A, Pedreschi D, Wang H (2013) Privacy-preserving mining of association rules from outsourced transaction databases. IEEE Syst J 7(3):385-395

Gkoulalas-Divanis A, Verykios VS (2009) Exact knowledge hiding through database extension. IEEE Trans Knowl Data Eng 21(5):699-713. http://doi.org/10.1109/TKDE.2008.199

Harnsamut N, Natwichai J (2008) A novel heuristic algorithm for privacy preserving of associative classification. In: PRICAI 2008: trends in artificial intelligence. Springer, Berlin, Heidelberg, pp 273-283

He Y, Siddharth B, Jeffrey FN (2011) Preventing equivalence attacks in updated, anonymized data. In: IEEE 27th international conference on data engineering (ICDE)

Ibrahim A, Jin H, Yassin AA, Zou D (2012) Towards privacy preserving mining over distributed cloud databases. In: IEEE second international conference on cloud and green computing (CGC)

Inan A, Saygin Y (2010) Privacy preserving spatio-temporal clustering on horizontally partitioned data. In: Lecture Notes in Computer Science (including Subseries Lecture Notes in Artificial Intelligence and Lecture Notes in Bioinformatics), 6202 LNAI. pp 187-198. http://doi.org/10.1007/978-3-642-16392-0_11

Islam MZ, Brankovic L (2011) Privacy preserving data mining: a noise addition framework using a novel clustering technique. Knowl Based Syst 24(8):1214-1223

Jain YYK, Yadav VKVVK, Panday GGS (2011) An efficient association rule hiding algorithm for privacy preserving data mining. Int J Comp Sci Eng 3(7):2792-2798 
Kamakshi P (2012) Automatic detection of sensitive attribute in PPDM. In: IEEE international conference on computational intelligence \& computing research (ICCIC)

Kamakshi P, Babu AV (2010) Preserving privacy and sharing the data in distributed environment using cryptographic technique on perturbed data 2(4)

Kantarcıoglu M, Vaidya J (2003) Privacy preserving naive bayes classifier for horizontally partitioned data. In: IEEE ICDM workshop on privacy preserving data mining, pp 3-9. http://www.cis.syr.edu/ wedu/ppdm2003/papers/1.pdf

Karim R, Rashid M, Jeong B, Choi H (2012) In: Transactional Databases. pp 303-319

Kasthuri S, Meyyappan T (2013) Detection of sensitive items in market basket database using association rule mining for privacy preserving. In: IEEE international conference on pattern recognition, informatics and mobile engineering (PRIME)

Kerschbaum F, Julien V (2008) Privacy-preserving data analytics as an outsourced service. In: Proceedings of the 2008 ACM workshop on secure web services. ACM

Keshavamurthy BN, Khan AM, Toshniwal D (2013) Privacy preserving association rule mining over distributed databases using genetic algorithm. Neural Comput Appl 22(S1):351-364

Kumbhar MN, Kharat R (2012) Privacy preserving mining of association rules on horizontally and vertically partitioned data: a review paper. In: 12th IEEE international conference on hybrid intelligent systems (HIS), pp 231-235

Lai J, Li Y, Deng RH, Weng J, Guan C, Yan Q (2014) Towards semantically secure outsourcing of association rule mining on categorical data. Inf Sci (Ny) 267:267-286

LiY (2013) Privacy-Preserving And Reputation System in Distributed Computing with Untrusted Parties, no. July. ProQuest LLC (2013). Copyright in the Dissertation held by the Author. Microform Edition @ ProQuest LLC. All rights reserved

Li W, Liu J (2009) Privacy preserving association rules mining based on data disturbance and inquiry limitation. In: 2009 Fourth International Conference on Internet Computer Science Engineering. pp 24-29

Li F, Ma J, Li J (2009a) Distributed anonymous data perturbation method for privacy-preserving data mining. J Zhejiang Univ Sci A 10(7):952-963

Li X, Liu Z, Zuo C (2009b) Hiding association rules based on relative-non-sensitive frequent itemsets. In: 2009 8th IEEE International Conference Cognitive Informatics. 384-389

Lin KW, LO Y-C (2013) Efficient algorithms for frequent pattern mining in many-task computing environments. Knowl Based Syst 49:10-21

Loukides G, Gkoulalas-divanis A (2012) Expert systems with applications utility-preserving transaction data anonymization with low information loss. Expert Syst Appl 39(10):9764-9777

Loukides G, Gkoulalas-Divanis A, Shao J (2012) Efficient and flexible anonymization of transaction data. Knowl Inf Syst 36(1):153-210

Machanavajjhala A, Kifer D, Gehrke J, Venkitasubramaniam M (2007) I-diversity: privacy beyond k-anonymity. ACM Trans Knowl Discov Data 1(1):3

Malina L, Hajny J (2013) Efficient security solution for privacy-preserving cloud services. In: 36th international conference on telecommunications and signal processing (TSP), pp 23-27. http://doi.org/10.1109/TSP.2013.6613884

Matwin S (2013) Privacy-preserving data mining techniques: survey and challenges. In: Discrimination and privacy in the information society. Springer, Berlin, Heidelberg, pp 209-221

Mokeddem D, Belbachir H (2010) Distributed classification using class-association rules mining algorithm. In: Proceedings of international conference on machine and web intelligence, ICMWI 2010, pp 334-337. http://doi. org/10.1109/ICMWI.2010.5647984

Monreale A, Pedreschi D, Pensa RG, Pinelli F (2014) Anonymity preserving sequential pattern mining. Artif Intell Law 22(2):141-173. http://doi.org/10.1007/s10506-014-9154-6

Mukkamala R, Ashok VG (2011) Fuzzy-based methods for privacy-preserving data mining. In: IEEE eighth international conference on information technology: new generations (ITNG)

Naeem M, Asghar S, Fong S (2010) Hiding sensitive association rules using central tendency. In: 6th international conference on advanced information management and service (IMS), pp 478-484. http://ieeexplore.ieee.org/xpls/abs_all. jsp?arnumber $=5713497$

Nanavati N, Jinwala D (2012) Privacy preservation for global cyclic associations in distributed databases. Procedia Technol 6:962-969

Nayak G, Devi S (2011) A survey on privacy preserving data mining: approaches and techniques. Int J Eng Sci Tech 3(3): 2117-2133

Nergiz ME, Gök MZ (2014) Hybrid k-anonymity. Comput Secur 44:51-63. http://doi.org/10.1016/j.cose.2014.03.006 Nergiz ME, Christopher C, Ahmet EN (2009) Multirelational k-anonymity. IEEE Trans Knowl Data Eng 21(8):1104-1117

Nergiz ME, Gök MZ, Özkanlı U (2013) Preservation of utility through hybrid k-anonymization. In: Trust, privacy, and security in digital business. Springer, Berlin, Heidelberg, pp 97-111

Nguyen XC, Le HB, Cao TA (2012) An enhanced scheme for privacy-preserving association rules mining on horizontally distributed databases. In: IEEE RIVF international conference on computing \& communication technologies, research, innovation, and vision for the future, pp 1-4. http://doi.org/10.1109/rivf.2012.6169821

Nix R, Kantarcioglu M, Han KJ (2012) Approximate privacy-preserving data mining on vertically partitioned data. In: Data and applications security and privacy XXVI. Springer, Berlin, Heidelberg, pp 129-144

Om Kumar CU, Tejaswi K, Bhargavi P (2013) A distributed cloud—prevents attacks and preserves user privacy. In: 15th international conference on advanced computing technologies, ICACT. http://doi.org/10.1109/ICACT.2013.6710509

Pan Y, Zhu X, Chen T (2012) Research on privacy preserving on K-anonymity. J Softw 7(7):1649-1656

Patel S, Garasia S, Jinwala D (2012) An efficient approach for privacy preserving distributed K-means clustering based on Shamir's, pp 129-141

Patil BB, Patankar AJ (2013) Multidimensional k-anonymity for protecting privacy using nearest neighborhood strategy. In: IEEE international conference on computational intelligence and computing research (ICCIC)

Qi X, Zong M (2012) An overview of privacy preserving data mining. Procedia Environ Sci 12(Icese 2011):1341-1347 
Quoc H, Arch-int S, Xuan H, Arch-int N (2013) Computers in industry association rule hiding in risk management for retail supply chain collaboration. Comput Ind 64(7):776-784

Raghuram B, Gyani J (2012) Privacy preserving associative classification on vertically partitioned databases. In: IEEE international conference on advanced communication control and computing technologies (ICACCCT), pp 188-192. http://doi.org/10.1109/ICACCCT.2012.6320768

Raju R, Komalavalli R, Kesavakumar V (2009) Privacy maintainance collaborative data mining: a practical approach. In: 2nd international conference on emerging trends in engineering and technology (ICETET), pp 307-311. http://doi. org/10.1109/ICETET.2009.184

Sachan A, Roy D, Arun PV (2013) An analysis of privacy preservation techniques in data mining. In: Advances in computing and information technology, vol 3. Springer, pp 119-128

Sathiyapriya K, Sadasivam GS (2013) A survey on privacy preserving association rule mining. Int J Data Min Knowl Manag Process 3(2):119-131

Seisungsittisunti B, Natwichai J (2011) Achieving k -anonymity for associative classification in incremental-data scenarios. In: Security-enriched urban computing and smart grid. Springer, Berlin, Heidelberg, pp 54-63

Shrivastava R, Awasthy R, Solanki B (2011) New improved algorithm for mining privacy_-preserving frequent itemsets. Int J Comp Sci Inform 1:1-7

Singh MD, Krishna PR, Saxena A (2010) A cryptography based privacy preserving solution to mine cloud data. In: Proceedings of third annual ACM Bangalore conference. ACM

Soodejani AT, Hadavi MA, Jalili R (2012) k-anonymity-based horizontal fragmentation to preserve privacy in data outsourcing. In: Data and applications security and privacy XXVI. Springer, Berlin, Heidelberg, pp 263-273

Sweeney L (2002) Achieving k-anonymity privacy protection using generalization and suppression. Int J Uncertain Fuzziness Knowl Based Syst 10(5):571-588

Tai C-H, Yu PS, Chen M-S (2010) k-Support anonymity based on pseudo taxonomy for outsourcing of frequent itemset mining. In: Proceedings of the 16th ACM SIGKDD international conference on knowledge discovery and data mining. ACM

Tai C-H, Huang J-W, Chung M-H (2013) Privacy preserving frequent pattern mining on multi-cloud environment. In: 2013 international symposium on biometrics and security technologies (ISBAST)

Tassa T (2014) Secure mining of association rules in horizontally distributed databases. IEEE Trans Knowl Data Eng 26(4):970-983. http://doi.org/10.1109/TKDE.2013.41

Vaidya J, Clifton C, Kantarcioglu M, Patterson AS (2008) Privacy-preserving decision trees over vertically partitioned data. ACM Trans Knowl Discov Data 2(3):1-27. http://doi.org/10.1145/1409620.1409624

Vatsalan D, Christen P, Verykios VS (2013) A taxonomy of privacy-preserving record linkage techniques. Inf Syst 38(6):946-969

Vijayarani S, Tamilarasi A, Seethalakshmi R (2010a) Privacy preserving data mining based on association rule: a survey. In: IEEE international conference on communication and computational intelligence (INCOCCI)

Vijayarani S, Tamilarasi A, Sampoorna M (2010b) Analysis of privacy preserving k-anonymity methods and techniques. In: IEEE international conference on communication and computational intelligence (INCOCCI), pp 540-545. http:// ieeexplore.ieee.org/xpls/abs_all.jsp?arnumber $=5738788$

Wang H, Hu C, Liu J (2010) Distributed mining of association rules based on privacy-preserved method. In: International symposium on information science and engineering (ISISE), pp 494-497. http://doi.org/10.1109/ISISE.2010.125

Wang ET, Lee G (2008) An efficient sanitization algorithm for balancing information privacy and knowledge discovery in association patterns mining. Data Knowl Eng 65(3):463-484

Wang K, Yu PS, Chakraborty S (2004) Bottom-up generalization: a data mining solution to privacy protection. In: IEEE Fourth international conference on data mining (ICDM'04)

Weng C, Chen S, Lo H (2008) A novel algorithm for completely hiding sensitive association rules. In: Eighth international conference on intelligent systems design and applications, ISDA'08, vol 3. pp 202-208. http://doi.org/10.1109/ ISDA.2008.180

Worku SG, Xu C, Zhao J, He X (2014) Secure and efficient privacy-preserving public auditing scheme for cloud storage. Comput Electr Eng 40(5):1703-1713

Xiong L, Chitti S, Liu L (2006) k nearest neighbor classification across. In: Proceedings of the 15th ACM international conference on information and knowledge management-CIKM'06, pp 840-841. http://doi. org/10.1145/1183614.1183757

Xu Z, Yi X (2011) Classification of privacy-preserving distributed data mining protocols. In: Sixth international conference on digital information management, pp 337-342. http://doi.org/10.1109/ICDIM.2011.6093356

Yi X, Zhang Y (2013) Equally contributory privacy-preserving k-means clustering over vertically partitioned data. Inf Syst 38(1):97-107

Ying-hua L, Bing-ru Y, Dan-yang C, Nan M (2011) State-of-the-art in distributed privacy preserving data mining. In: IEEE 3rd international conference communication software and networks, pp 545-549. http://doi.org/10.1109/ ICCSN.2011.6014329

Zhang G, Yang Y, Chen J (2012a) A historical probability based noise generation strategy for privacy protection in cloud computing. J Comput Syst Sci 78(5):1374-1381. http://doi.org/10.1016/j.jcss.2011.12.020

Zhang G, Zhang X, Yang Y, Liu C, Chen J (2012b) An association probability based noise generation strategy for privacy protection in cloud computing. 639-647

Zhang X, Liu C, Yang C, Dou W, Chen J (2013a) Combining top-down and bottom-up : scalable sub-tree anonymization over big data using MapReduce on cloud

Zhang X, Liu C, Nepal S, Chen J (2013b) An efficient quasi-identifier index based approach for privacy preservation over incremental data sets on cloud. J Comput Syst Sci 79(5):542-555. http://doi.org/10.1016/j.jcss.2012.11.008

Zhang X, Liu C, Chen J (2013c) Journal of computer and system sciences an efficient quasi-identifier index based approach for privacy preservation over incremental data sets on cloud. J Comput Syst Sci 79(5):542-555 
Zhang X, Liu C, Nepal S, Yang C, Dou W, Chen J (2014a) A hybrid approach for scalable sub-tree anonymization over big data using MapReduce on cloud. J Comput Syst Sci 80(5):1008-1020

Zhang X, Yang LT, Member S, Liu C, Chen J (2014b) A scalable two-phase top-down specialization approach for data anonymization using MapReduce on cloud. 25(2): 363-373

Zhu X, Chen T (2012) Research on privacy preserving based on k-anonymity. In: Computer, informatics, cybernetics and applications. Springer, Netherlands, pp 915-923

Submit your manuscript to a SpringerOpen ${ }^{\circ}$ journal and benefit from:

- Convenient online submission

- Rigorous peer review

- Immediate publication on acceptance

- Open access: articles freely available online

- High visibility within the field

- Retaining the copyright to your article

Submit your next manuscript at $>$ springeropen.com 